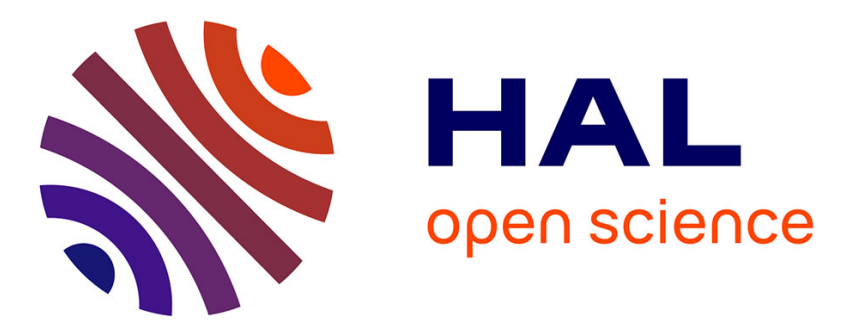

\title{
On the use of spectral methods for the numerical solution of stiff problems
}

\author{
Hervé Guillard, Roger Peyret
}

\section{To cite this version:}

Hervé Guillard, Roger Peyret. On the use of spectral methods for the numerical solution of stiff problems. [Research Report] RR-0553, INRIA. 1986. inria-00076001

\section{HAL Id: inria-00076001 https://hal.inria.fr/inria-00076001}

Submitted on 24 May 2006

HAL is a multi-disciplinary open access archive for the deposit and dissemination of scientific research documents, whether they are published or not. The documents may come from teaching and research institutions in France or abroad, or from public or private research centers.
L'archive ouverte pluridisciplinaire HAL, est destinée au dépôt et à la diffusion de documents scientifiques de niveau recherche, publiés ou non, émanant des établissements d'enseignement et de recherche français ou étrangers, des laboratoires publics ou privés. 


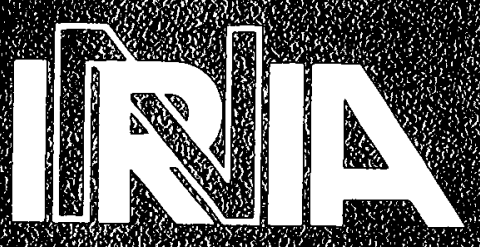

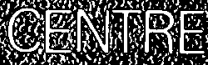

SO1:

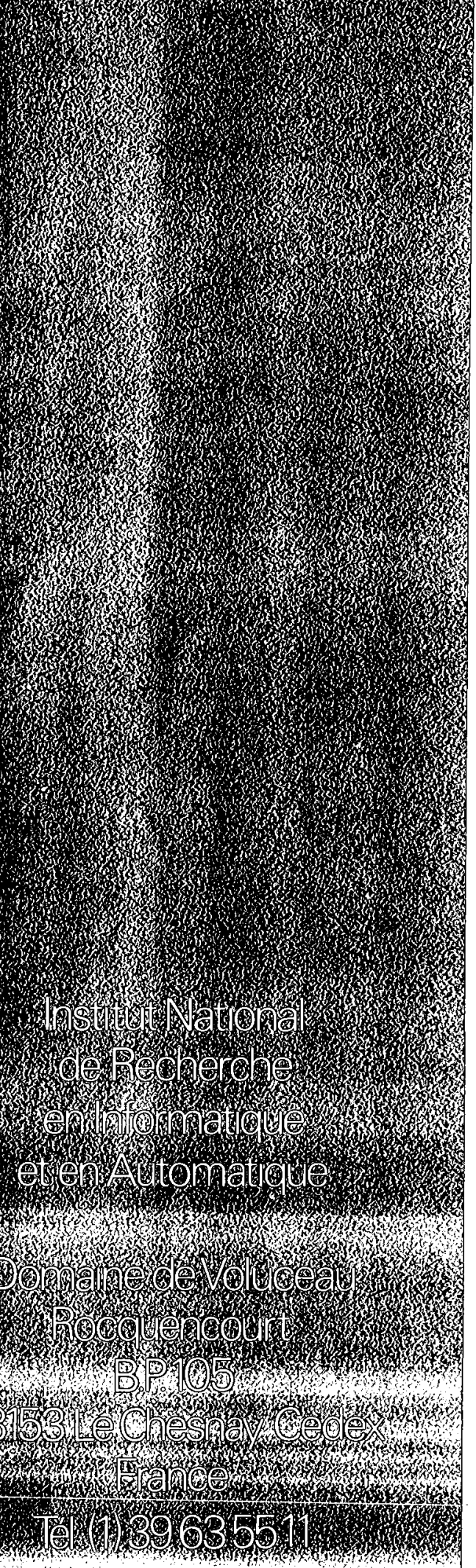

\%

3.

. 201

3.

16.

s. or 3

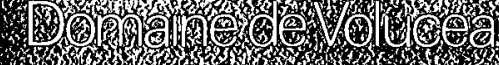

70 .

13. 7.7.6.

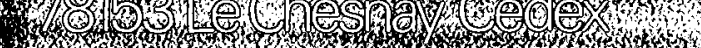
36.

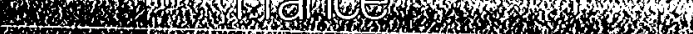

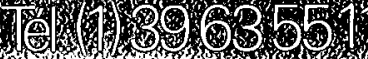

\section{Rapports de Recherche}

$\mathrm{N}^{\circ} 553$

\section{ON THE USE OF SPECTRAL METHODS FOR THE NUMERICAL SOLUTION OF STIFF PROBLEMS}

Hervé GUILLARD

Roger PEYRET

Juillet 1986 


\section{GUILLARD - PEYRET}

"On the use of Spectral methods for the numerical solution of stiff problems" .

titre en francais: "Sur l'emploi des méthodes spectrales pour la résolution numérique de problèmes "raides".

\section{RESUME EN FRANCGAIS :}

Ce rapport traite de certains aspects de l'emploi de méthodes spectrales pour la résoiution numérique de systèmes d'équations aux dérivées partielles "raides". On montre que, malgré leur haute précisicr. ces méthodes nécessitent d'utiliser un grand nombre de modes pour obter ${ }^{\circ}$. une solution suffisamment précise. Dans ces conditions, il est préféráb: de considérer une sorte de "maillage" adapté. On présente une méthode de construction d'un tel maillage fondée sur un changement de variable déterminé à partir du calcul de la norme de la solution. Divers exemples numériques sont présentés et discutés. 


\title{
ON THE USE OF SPECTRAL METHODS FOR THE NUMERICAL SOLUTION OF STIFF PROBLEMS
}

\author{
Hervé Guillard ${ }^{\dagger}$ and Roger Peyret $\ddagger$
}

TINRIA, Sophia Antipolis, 06560 Valbonne, France

$\$$ Dpt of Mathematics, Nice University, Parc Valrose, 06000 Nice, France and INRIA, Sophia Antipolis, 06560 Valbonne, France

\begin{abstract}
This report describes some aspects of the use of spectral methods for the numerical solution of systems of stiff partial differential equations. It is shown that despite the high spatial precision of these methods, a reasonnable accuracy can only be attained with a large number of modes and therefore, some kind of adaptive "gridding" is necessary. A way to implement this adaptation, based on the computation of a norm of the solution is proposed. Numerical examples are
presented and discussed.
\end{abstract}




\section{INTRODUCTION}

In the past few years, a large number of methods have been proposed to overcome the practical difficulties posed by the numerical solution of systems of stiff ordinary differential equations (ODE). One key of the success of these methods lies in the automatic control of the time step that is assuring the opposite requirements of stability and accuracy. Let us recall that in ODE systems the term of stiffness is associated with the presence of relaxation times of very disparate magnitude. Extending this definition to the context of partial differential equations (PDE) we shall consider stiffness for a system of equations that presents a great disparity in both the time and space characteristic scales of the problem . For example, if we consider the propagation of a plane flame front in a $1 \mathrm{~m}$ long closed tube, in a calculation coupling hydrodynamics with chemical kinetics, we shall have to deal with the following time scales :

(1) chemical time scales of order $10^{-6} \mathrm{sec}$

(2) characteristic time of acoustic waves of order $10^{-9} \mathrm{sec}$

(3) sound transit time of $10^{-3} \mathrm{sec}$

(4) flame transit time of $1 \mathrm{sec}$ (with a typical flame speed of $1 \mathrm{~m} / \mathrm{sec}$ )

and with the following space scales :

(1) flame inner zone of order $10^{-3} \mathrm{~cm}$

(2) flame thickness of order $10^{-2} \mathrm{~cm}$

(3) diffusion scale of $10^{-1} \mathrm{~cm}$

(4) convective scale of $10 \mathrm{~cm}$

(5) dimension of the tube $100 \mathrm{~cm}$ 
If complex chemistry is present, we also have to consider simultaneous chemical species profiles with high spatial gradients of different magnitudes, each with its own propagation velocity. Clearly the solution of such a problem, that we define as a stiff PDE problem, represents a formidable task for numerical analysis and requires the development of specific methods to handle the difficulties associated with these different scales.

Extending to the spatial domain the automatic time step control that proves to be very efficient in the numerical solution of ODE systems, one of the most interesting solution provided for stiff PDEs is to control the space mesh size and to distribute the grid points in relation to the evolution of the calculated solution. The different tehniques for moving the nodes and the selection of criteria to dynamically adapt the grid to the solution are probably one of the most important areas of research in the development of solvers for stiff PDE systems. These criteria may be of different types, one of the most popular consists in constructing a fonctionnal that is minimum when some requirements are satisfied. For example, items of concern are the concentration of the nodes in the regions with high spatial derivatives, ([10], [18]), the smoothness or the orthogonality of the grid ([3],[30]). Lagrangian approaches where the nodes are moved according to a prescribed velocity $([27])$ and some more sophisticated methods such as the moving finite element $([21],[22],[12])$ also proved to be efficient in this context.

Applications of the previous techniques to spectral methods are only at a very early stage of development and only some authors have presented some results relevant to this topic. Lacroix \& al in [1] have presented a solution of Burgers' equation based on a Chebyshev expansion and on a procedure to concentrate the grid points in the region where the solution exhibits a large gradient. Brachet [2]in a shear flow problem used a similar technique and Hussaini \& Zang [16] have applied an adaptive coordinate transform to follow a shock in a gas dynamics problem. In fact the transposition to spectral approximations of the methods of adaptive gridding is usually done via a coordinate transform which changes the original stiff problem posed in the physical space to a simpler one posed in the computational domain, this procedure is very similar to that proposed by Dwyer \& Sanders [10] for finite difference methods. However, none of these authors have proposed a general method to solve these problems of adaptation and it is the purpose of this work to investigate a general procedure of adaptation suitable for spectral approximations. Note also that coordinate transforms are frequently used in spectral methods (see Orszag [24] or Morchoisne [23]), however the procedure is usually employed to match a complex geometry and so is not relevant to our subject.

Suppose the solution of the problem under interest is smooth $\left(C^{\infty}\right)$, owing to the exponential rate of convergence of spectral methods, one may ask if adaptive techniques are actually a necessity for this class of numerical techniques. However, exponential rate of convergence is only achieved in spectral computations when the number of retained modes is sufficiently high and this number can be prohibitive in practice and the use of a special technique to keep the computational cost into reasonable bounds is often necessary. 
As spectral methods are essentially Weighted Residuals Methods, the distance between the exact and the discrete solutions is bounded by the projection error (the distance between the solution and its orthogonal projection) or if a pseudo-spectral (collocation) method is used, by the interpolation error (the distance between the solution and its interpolated polynomial). So, let us recall some known results about polynomial interpolation. The following will concentrate upon Chebyshev interpolation as the numerical examples presented through this paper use Chebyshev methods, however similar results exist for Fourier methods (see Pasciak [26], Mercier [20] and Kreiss \& Oliger [17]) or for Legendre spectral methods (see Canuto \& Quarteroni [5] and Maday \& Quarteroni [19]).

We put the following definition :

$\mathbf{L}_{\omega}^{2}$ is the set of functions from $[-1,+1]$ to $\mathbf{C}$ square integrable for the inner product :

$$
(\varphi, \psi)_{\omega}=\int_{-1}^{+1} \varphi(x) \overline{\psi(x)} \omega(x) d x
$$

where $\omega(x)=\left(1-x^{2}\right)^{-\frac{1}{2}}$ is the Chebyshev weight function.

For any integer number $m$, we also define the Sobolev space $\mathbf{H}_{\omega}^{m}$ as :

$$
\mathbf{H}_{\omega}^{m}=\left\{u: u^{(\alpha)} \in \mathbf{L}_{\omega}^{2}, \alpha=0, \ldots, m\right\}
$$

This space is a Hilbert space for the norm :

$$
\|u\|_{m, \omega}=\left(\sum_{\alpha=0}^{m}\left\|u^{(\alpha)}\right\|_{\omega}^{2}\right)^{1 / 2}
$$

We denote by $\mathbf{P}_{N}$ the space of the polynomials of degree $\leq N$ and by $P_{N}$ the orthogonal projection from $\mathbf{L}_{\omega}^{2}$ to $\mathbf{P}_{N}$.

We then have the following theorem (see Canuto \& Quarteroni [5] for the proof) :

For any $\mu$ and $\sigma \geq 0$ and any $u \in \mathbf{H}_{\omega}^{\sigma}$ we have the estimate :

$$
\left\|u-P_{N} u\right\|_{\mu, \omega} \leq C\|u\|_{\sigma, \omega} \begin{cases}N^{\frac{3 \mu}{2}-\sigma} & 0 \leq \mu \leq \min (1, \sigma) \\ N^{2 \mu-\frac{1}{2}-\sigma} & 1 \leq \mu \leq \sigma\end{cases}
$$

where $C$ is a constant independent of $N$.

In a analogous manner, if we define $P_{C}$ as the interpolation operator associated with the Gauss-Lobatto-Chebyshev collocation points $\xi_{j}$ :

$$
\xi_{j}=\cos \frac{j \pi}{N}, j=0, \cdots, N \text {. }
$$


The following estimate holds :

$$
\left\|u-P_{C} u\right\|_{\mu, \omega} \leq C N^{2 \mu-\sigma}\|u\|_{\sigma, \omega}
$$

for any $\sigma \geq 1 / 2$, any $0 \leq \mu \leq \sigma$ and any $u \in \mathbf{H}_{\omega}^{\sigma}$.

Suppose we intend to solve with a spectral or pseudo-spectral method a problem of the form :

$$
\begin{cases}\frac{\partial}{\partial t} u+L u=f, & \text { in } \Omega \\ B(u)=0 & \text { on } \partial \Omega\end{cases}
$$

where $L$ is a spatial differential operator and $B$ is some boundary operator.

As mentioned earlier, we can expect that the approximation error will be bounded by the projection or interpolation error. Hence, due to the above estimates (1.4) and (1.6) the approximation error will be bounded by an expression of the form :

$$
C N^{e(\sigma, \mu)}\|u\|_{\sigma, \omega}
$$

where $e(\sigma, \mu)$ is one of the expressions appearing in theorem (1.4) or (1.6). Now, suppose, as is common in the context of stiff PDE's, that the norm of the solution (supposed to be $\left.C^{\infty}\right)$ satisfies an equality of the form :

$$
C_{\sigma+1}\|u\|_{\sigma+1, \omega} \geq M C_{\sigma}\|u\|_{\sigma, \omega}
$$

where $M$ is a fairly large number, then one can see that an exponential rate of convergence (that is the rate of convergence expected from spectral methods) can only be retrieved if $N \geq M$. This requirement can be a very severe one. For example, dealing with the unsteady Burgers' equation, Lacroix \& al [1] find necessary to use at least 512 Chebyshev polynomias to get a satisfactory solution for the problem considered in Section 3.4.

Hence, clearly there is a need of some special procedure to handle this kind of equations. In the following Section we investigate one of the possible solution to this problem.

\section{ADAPTIVE PROCEDURE FOR SPECTRAL METHODS}

Considering a stiff PDE problem whose solution verifies an inequality of the form $C_{\sigma+1}\|u\|_{\sigma+1, \omega} \geq M C_{\sigma}\|u\|_{\sigma, \omega}$ we have seen in the previous Section that we can recover an exponential rate of convergence for our spectral approximation of problem (1.7) only if the number of modes retained in this approximation is greater or equal to $M$. In a large number of situations of practical interest, this can be an untolerable constraint and we have to find a special procedure to deal with these kind of problems. In finite difference or finite element methods the solution is to redistribute the grid points where they are the 
most needed and one can look for an analogous procedure suitable for spectral methods. However, note that we have not the same possibility to move one node independently from the others when using spectral methods. On the other hand in principle, pseudo-spectral methods may allow us to definite collocation points in an arbitrary manner, therefore these methods should have the same abilities than finite difference or finite element methods, however the rate of convergence of a polynomial interpolation is highly dependent of the collocation network. Recall for example that the convergence of polynomial interpolation is rather poor on a regular network. So it is our belief that such a method though possible should be better grounded in theoretical results on polynomial interpolation; Known results are today quite partial (Some theoretical and numerical examples of such results may be found in Durand [9]).

Another possibility which permits to keep the usual distribution of the collocation points, is to perform a coordinate transform and to associate problem (1.7) with the modified problem :

$$
\begin{cases}\frac{\partial}{\partial t} v+L^{*} v=f^{*}, & \text { in } \Omega^{*} \\ B^{*}(v)=0 & \text { on } \partial \Omega^{*}\end{cases}
$$

where $\Omega^{*}$ is the computationnal space, $L^{*}$ and $B^{*}$ the transformed operators, $v$ the transformed solution and $f^{*}$ the transformed source term.

We want the transformed system (2.1) to be less stiff than the original one ( 1.7 ) and therefore the problem is now to choose the coordinate transform :

$$
\begin{aligned}
& \Omega^{*} \underset{\mathrm{g}}{\longrightarrow} \Omega \\
& \xi \stackrel{\mathrm{b}}{\longmapsto} \mathrm{x}
\end{aligned}
$$

to satisfy this requirement.

In the works above [1],[2], the fonction $\mathrm{g}$ is given a priori to concentrate the nodes in the regions of interest. Such a procedure requires an a priori knowledge of some features of the solution such as the location of regions of high gradients, their propagation velocity etc ..., Furthermore, because $g$ is not time dependent this procedure is not applicable when the solution is evolving spatially during the course of the computation.

So, this solution is not very general and we have to find another technique which requires no a priori knowledge of the solution and is applicable to problems with rapidly evolving solutions as well as stationary ones. Returning to the majorations of the estimates (1.4) and (1.6) one can see that for a fixed number of modes, the better the solution is approximated by its projection or interpolation polynomial, the smaller is its norm. This leads us to search for the coordinate transform $\mathrm{g}$ as the transform which minimizes a certain norm of the solution. Therefore the original problem (1.7) is now replaced by : 
Find $v$ and $g$ such that :

$$
\begin{cases}\frac{\partial}{\partial t} v+L^{*} v=f^{*}, & \text { in } \Omega^{*} \\ B^{*}(v)=0 & \text { on } \partial \Omega^{*} \\ \text { and } g \text { is such that a certain norm, } & \\ \text { say }\|v\|_{\nu, \omega} \text { of the solution is minimum. } & \end{cases}
$$

where $\nu=1,2, \ldots$. Though more complicated, this procedure is quite general and can be use $\bar{d}$ in cases where the structure of the solution moves rapidly inside the whole domain. The minimization of the $H_{\omega}^{\nu}$ norm of the solution $\|u\|_{\nu, \omega}$ has its equivalence in some methods of adaptation proposed for finite difference methods, consider for example the meaning of the minimization of $\|u\|_{1, \omega}$ :

Find $\mathrm{x}=\mathrm{g}(\xi)$ such that :

$$
J(g)=J\left(x, x_{\xi}\right)=\int_{-1}^{+1}\left(u^{2}+u_{x}^{2} x_{\xi}^{2}\right) \omega(\xi) d \xi \text { is minimum }
$$

Since the value of the function $u$ itself is of no use in the minimization of $\mathrm{J}(\mathrm{g})$ we may change the definition of the functional to :

$$
J(g)=\int_{-1}^{+1}\left(u_{x}^{2} x_{\xi}^{2}\right) \omega(\xi) d \xi
$$

The Euler equation corresponding to this problem writes :

$$
-\left(u_{x} u_{x x} x_{\xi}^{2}-u_{x}^{2} x_{\xi \xi}\right) \omega(\xi)+u_{x}^{2} x_{\xi} \frac{d \omega(\xi)}{d \xi}=0
$$

The last term of the left-hand side of this expression is due to the use of Chebyshev polynomials and emphasizes the special role played by the boundaries in this spatial approximation while in the first one we can recognize a term which in the frame of finite difference methods has the effect of concentrating the grid nodes in the regions of steep gradients. In fact, if (2.6) is reduced to this term, it admits the following first integral :

$$
u_{x}^{2} x_{\xi}^{2}=c t e
$$

which imposes that the number of grid points in a region is proportional to the gradient of $u$.

The use of higher order norms follows the same idea, so the use of the $H_{\omega}^{2}$ norm will involve a combination of the first and second derivatives of $u$ and will concentrate the collocation points in regions where these quantities are large. The question arises of what should be the order of the employed Sobolev norm. Note that due to the dependence on $\sigma$ of the constant $C_{\sigma}$ appearing in expressions (1.4) and (1.6) the higher norm is not necessarily the 
better. Moreover the computation of high norms can become rather lengthy and solving the associated minimization problem rather expensive. Our computational experience show us that the recourse to the $H_{\omega}^{2}$ norm is sufficient and that the use of the $H_{\omega}^{3}$ norm does not significantly improve the results for the problems we solved with this technique.

Let us now examine the ways of solving the minimization problem associated with equations (2.3). This problem is not an easy one. There are two difficulties associated with it: 1) we want the coordinate transform $g$ to be known at each point in the domain of computation and 2) to maintain the spatial accuracy of the spectral method, we want it to be a sufficiently smooth function. A possible way is to solve numerically the Euler PDE problem associated with (2.3) (this possibility is rather frequently utilized in grid generation techniques, see Thompson [30]). However this Euler equation can be a rather complicated (and non-linear) one if we use an $H_{\omega}^{\sigma}$ norm with $\sigma>1$. Hence, we have to turn ourselves to optimisation techniques.

The solution we have retained here is to choose the coordinate transform $\mathrm{g}$ as a parametrized function whose analytical form is known a priori. This will allow us to transform the minimization problem in (2.3) into an optimisation problem on the finite dimension space of the parameters. Although not entirely satisfactory, this procedure was chosen as a preliminary step to test the capabilities of the proposed method of adaptation. Note also that this procedure is rather general as the parameters may be the coefficients of a set of basis functions.

However, there is an additional problem associated with the definition of the coordinate transform $\mathrm{g}$ : As will be explained in the examples of Section 3 if we intend to adapt continuously the grid to the evolving solution we need to invert the coordinate transform as we have to project at each time step the old solution on a new grid, so there is a possibility of a loss of information if this inversion is not exact and this can affect the accuracy of the approximation.

In the examples given later the inversion of $\mathrm{g}$ is always known analytically but in the general case this could lead to some difficulties and this point requires further investigation.

The procedure described above may be used in two different ways according to the characteristics of the expected solution: When the solution is stationary or although not stationary, is evolving in a limited region and since in this case a fixed coordinate transform may be sufficient, this procedure may be thought as a way to find an optimal or near optimal coordinate transform. Then one simply has to compute the $H_{\omega}^{\sigma}$ norm of the solution (in the computationnal space) and to rely on it to find the best coordinate transform. When the physical phenomenon of interest is rapidly moving inside a large region of the domain, a fixed coordinate transform may not be sufficient and we have to adapt it continuously to the evolving solution. The procedure described above was mainly designed for this case. Then one has to compute at each time step the $H_{\omega}^{\sigma}$ norm of the solution (in the computational space) and to find the coordinate transform that minimizes this norm. The following Section will give some examples of the two ways to use the procedure which has been described in this Section. 


\section{NUMERICAL EXAMPLES.}

Since the numerical examples presented in this Section make use of the collocation (or pseudo-spectral) method, we shall begin with recalling the outlines of this method.

\subsection{The collocation method}

Suppose we have to solve an evolution problem of the form :

$$
\begin{cases}\frac{\partial}{\partial t} u+L u=f, & \text { in } \Omega \\ B(u)=0 & \text { on } \partial \Omega \\ u(x, 0)=h(x) & \end{cases}
$$

where $u, h$ and $f$ belong to a Hilbert space $H, L$ is an operator on this space and $B$ is a boundary operator. The collocation approximation of problem (3.1.1) involves the definition of a finite dimensional subspace $S_{N}$ of $H$, a collocation network $\left\{x_{i}\right\}_{i=1, \ldots, N}$ of points of $\Omega \cup \partial \Omega$ and of an interpolation operator $\mathbf{P}_{\mathbf{C}}$ from $H$ to $S_{N}$. This interpolation operator $\mathbf{P}_{\mathbf{C}}$ is defined by :

$$
\left\{\begin{array}{l}
\mathbf{P}_{\mathrm{C} u} \in S_{N} \\
\mathbf{P}_{\mathrm{C} u\left(x_{i}\right)=u\left(x_{i}\right), i=1, \ldots, N}
\end{array}\right.
$$

Then we substitute to problem (3.1.1) the following approximate problem :

$$
\begin{cases}\frac{\partial}{\partial t} u_{N}+L_{N} u_{N}=f_{N}, & \text { in } \Omega \\ B_{N}\left(u_{N}\right)=0 & \text { on } \partial \Omega \\ u_{N}(x, 0)=\mathbf{P}_{C} h & \end{cases}
$$

where $u_{N} \in S_{N}, L_{N}=\mathbf{P}_{\mathrm{C}} L \mathbf{P}_{\mathrm{C}}$ and $f_{N}=\mathbf{P}_{\mathrm{C}} f$. Since there is a one-to-one correspondence between a vector $\mathbf{a}_{N}=\left(a\left(x_{1}\right), \ldots, a\left(x_{N}\right)\right)^{t}$ and a function $a_{N}$ of $S_{N}$, problem (3.1.3) is equivalent to the following system of $O D E$ :

$$
\left\{\begin{array}{l}
\frac{d}{d t} \mathbf{u}_{N}+A \mathbf{u}_{N}=\mathbf{f}_{N} \\
\mathbf{u}_{N}(0)=\mathrm{h}_{N}
\end{array}\right.
$$

where $\mathbf{A}$ is the vector function corresponding to the operator $L_{N}$. This function is such that if the operator $L$ is linear;

$$
\mathbf{A u}=\mathbf{D u}
$$


where $\mathbf{D}$ is an NxN matrix. The form of this matrix for the usual operators $\frac{\partial}{\partial x}$ or $\frac{\partial^{2}}{\partial x^{2}}$ can be found for example in Fornberg [11] for the Fourier collocation method and in Ouazzani \& Peyret [25] and Badesvant et al [1] for the Chebyshev collocation method and their properties are studied in Gottlieb \& Orszag [14], Gottlieb \& Lustman [13], and Canuto \& Quarteroni [6].

When the operator $\mathrm{L}$ is non-linear or linear with variable coefficients, collocation method are much simpler to implement than spectral method.

Consider for example the treatment of a term of the form $a(x) \frac{\partial}{\partial x}$ in the Fourier method. The spectral treatment of such a term will involve a convolution product of the form :

$$
i\left(\sum_{p=-K}^{K} \sum_{q=-K}^{K} q \hat{a}_{p} \hat{u}_{q}\right) \quad p+q=k
$$

which is expensive to compute, on the contrary this term in a collocation method simply writes $\mathbf{A D u}$ where $\mathbf{A}$ is a diagonal matrix whose entries are $\mathrm{a}\left(x_{i}\right), \mathbf{D}$ is the Fourier derivation matrix and $u$ is the vector of the value of $u$ at the collocation points $x_{i}$. Note that the computation of the product Du may be saved thanks to the pseudospectral technique consisting in performing the differentiation in the spectral space and in using FFT algorithm to link spectral and physical spaces. We do not discuss here the stability and accuracy of these different methods, for a study of these questions we refer to [14] or from a more fundamental point of view to Mercier [20]. The examples given in the following Section will give more examples of the treatment of non-linear terms in collocation methods.

\subsection{Stationary spherical flame.}

\subsubsection{The physical problem.}

In our first example we are interested in a premixed spherical flame stabilized by a point source of combustible mixture. This system is described by the following set of equations:

$$
\begin{aligned}
& \frac{\partial C}{\partial t}+\frac{\kappa}{r^{2}} \frac{\partial C}{\partial r}=\frac{1}{L} \frac{1}{r^{2}} \frac{\partial}{\partial r}\left(r^{2} \frac{\partial C}{\partial r}\right)+\omega \\
& \frac{\partial T}{\partial t}+\frac{\kappa}{r^{2}} \frac{\partial T}{\partial r}=\frac{1}{r^{2}} \frac{\partial}{\partial r}\left(r^{2} \frac{\partial T}{\partial r}\right)+\omega
\end{aligned}
$$

$C$ is the dimensionless concentration of combustion product; $T$ is the dimensionless temperature defined as the ratio $\frac{T-T_{0}}{T_{b}-T_{0}}$ where $T_{0}$ is the fresh gas temperature and $T_{b}$ is 
the adiabatic temperature of combustion; $L$ is the Lewis number ; $r$ is the dimensionless radial coordinate referred to the flame thickness ; $4 \pi \kappa$ is the mass flux of the source and $\omega$ is the reaction rate which is of Arrhenius form :

$$
\omega=\frac{1}{2 L} \beta^{2}(1-C) \exp (\beta(T-1))
$$

$\beta$ is the dimensionless activation energy referred to $R T_{b}$ where $R$ is the universal gas constant.

In the fresh mixture, far ahead from the flame, the temperature of the gas is equal to the temperature of the fresh mixture $T_{0}$ while the concentration of the burnt gas is zero. In the burnt gas, beyond the flame, we assume that the reaction is complete, so the temperature is equal to $T_{b}$ and the concentration to $C_{b}$. Therefore in dimensionless variables, we have the following boundary conditions :

$$
\begin{gathered}
T \rightarrow 0, C \rightarrow 0 \text { as } r \rightarrow 0 \\
T \rightarrow 1, C \rightarrow 1 \text { as } r \rightarrow+\infty
\end{gathered}
$$

The asymptotic analysis of the previous problem by Sivashinsky [29], shows that a stationary solution exists for each $\kappa$ such that the radius of the flame (equal in this case, to first order, to $\sqrt{\kappa}$ ) is greater than the flame thickness.

\subsubsection{Numerical method.}

The system of equations (3.2.1)-(3.2.2) is approximated by a Chebyshev collocation procedure. As Chebyshev polynomials are defined in $[-1,+1]$; we have to perform a coordinate transform to map $[-1,+1]$ into $[0,+\infty]$. The question of the mapping of $[-1,+1]$ into an infinite domain is discussed in Grosch \& Orszag [15] and in Boyd [4]: They find that the convergence of Chebyshev polynomials is good when ah algebraic mapping or a domain truncation are performed but that this convergence is slowed down when an exponential mapping is used *. In this paragraph, a domain truncation is done, then the problem (3.2.1)-(3.2.2) is solved in an interval $\left[r_{0}, r_{\infty}\right]$ where $r_{0}$ and $r_{\infty}$ are respectively small and large enough. Near the flame radius, the solution exhibits a large gradient therefore the transform is also chosen as to concentrate the collocation points in this region. A coordinate transform of the following form was chosen to fulfill this requirement (see appendix I) :

$$
\begin{aligned}
& {[-1,+1] } \longrightarrow\left[r_{0}, r_{\infty}\right] \\
& \xi \quad \stackrel{\mathrm{g}}{\longrightarrow} r=a+b e^{-\lambda \xi}-c e^{\nu \xi}
\end{aligned}
$$

* Nóte, huwever that Hussaini \& Zang [17] have used exponential mapping with success. 
In the computational space the basic equations, (3.2.1) and (3.2.2) can be written as :

$$
\begin{aligned}
& \frac{\partial C}{\partial t}+\frac{\kappa}{r^{2} r_{\xi}} \frac{\partial C}{\partial \xi}=\frac{1}{L} \frac{1}{r^{2} r_{\xi}} \frac{\partial}{\partial \xi}\left(\frac{r^{2}}{r_{\xi}} \frac{\partial C}{\partial \xi}\right)+\omega \\
& \frac{\partial T}{\partial t}+\frac{\kappa}{r^{2} r_{\xi}} \frac{\partial T}{\partial \xi}=\frac{1}{r^{2} r_{\xi}} \frac{\partial}{\partial \xi}\left(\frac{r^{2}}{r_{\xi}} \frac{\partial T}{\partial \xi}\right)+\omega
\end{aligned}
$$

and the boundary conditions (3.2.4) and (3.2.5) are replaced by :

$$
\begin{aligned}
& T=C=0 \text { as } \xi=-1 \\
& T=C=1 \text { as } \xi=+1
\end{aligned}
$$

These equations are solved in the physical space by a Chebyshev collocation method : For example, defining $\mathbf{C}$ as the vector $\left(C_{0}, \ldots, C_{N}\right)^{t}$ where $C_{i}$ is the value of the concentration at the collocation point $\xi_{i}$ defined by (1.5), equation (3.2.7) takes the form :

$$
\frac{\partial \mathbf{C}}{\partial t}+E D \mathbf{C}=F D \mathbf{C}+G D^{2} \mathbf{C}+\omega
$$

where $E, F, G$ are the diagonal matrices whose entries are respectively :

$$
\frac{\kappa}{r^{2}\left(\xi_{i}\right) r_{\xi}\left(\xi_{i}\right)}, \quad\left(\frac{2}{r\left(\xi_{i}\right) r_{\xi}\left(\xi_{i}\right)}-\frac{r_{\xi \xi}}{\left(r_{\xi}\left(\xi_{i}\right)\right)^{3}}\right) \text { and } \frac{1}{r_{\xi}^{2}\left(\xi_{i}\right)} ;
$$

$D$ is the Chebyshev derivation matrix and $\omega$ is the vector $\left(\omega\left(\xi_{0}\right), \ldots, \omega\left(\xi_{N}\right)\right)^{t}$.

One can see that in this method, the computation of the linear terms with variable coefficients reduce to matrix multiplications that are easily performed on modern vector computer, while if a spectral method were used the computation of these terms would be rather expensive and the computation of the non-linear term $\left(\omega\left(\xi_{0}\right), \ldots, \omega\left(\xi_{N}\right)\right)^{t}$ would become cumbersome.

A two-time level scheme is used to integrate in time equation (3.2.11)(and the analogous temperature equation); it writes :

$$
\begin{gathered}
{\left[I-\theta \delta t\left(E D-F D-G D^{2}\right)\right]\left(C^{n+1}-C^{n}\right)=} \\
\delta t \omega^{n}+\delta t\left[F D+G D^{2}-E D\right] C^{n}
\end{gathered}
$$

where $I$ is the identity matrix and $\delta t$ is the time step.

Since we are only interested in the stationary solution the value $\theta=1$ corresponding to an implicit scheme for the linear term of equation (3.2.11) was retained for the example presented below. This scheme is first order in time and it is unconditionnaly stable for the linear part of the equations, a constraint on the time step based on the maximum value of the production term is imposed to ensure the stability of the global discretisation.

The boundary conditions are explicitely implemented in equation (3.2.12) : i.e the first 
and last lines of the linear system (3.2.12) are replaced by the boundary conditions. Since the resulting matrix does not depend on time it can be inverted once for all at the beginning of the computation and therefore the time-marching procedure is simply performed in two steps :

1) compute the right hand side of (3.2.12) (this can be efficiently done with FFT algorithms) and

2) multiply it by $\left[I+\theta \delta t\left(E D-F D-G D^{2}\right)\right]^{-1}$

\subsubsection{Numerical results}

We present below the results obtained for the following values of the parameters : $\kappa=$ $10475, \beta=10$ and $L=1$. The initial condition is the asymptotic stationnary solution of equations (3.2.1) and (3.2.2), it writes :

$$
\begin{cases}T(r)=\exp \left(\frac{\kappa}{r_{f}}-\frac{\kappa}{r}\right), & \text { if } r \leq r_{f} \\ T(r)=1, & \text { if } r \geq r_{f}\end{cases}
$$

and

$$
\begin{cases}C(r)=\exp \left(L\left(\frac{\kappa}{r_{f}}-\frac{\kappa}{r}\right)\right), & \text { if } r \leq r_{f} \\ C(r)=1, & \text { if } r \geq r_{f}\end{cases}
$$

where $r_{f}$ is the radius of the flame, obtained from an asymptotic analysis including terms up to $1 / \beta$, analogous to the one proposed by Deshaies et al [8]. In this example we take $r_{f}=93.4$, the values of the parameters defining the coordinate transform are reported in Appendix I.

Note that the derivative of the initial condition is not definite at $r=r_{f}$. A discontinuity in the initial solution or in its derivatives may sometimes bring problems when a spectral method is used. However we do not experience difficulties with it though the convergence of the Chebyshev series to this function is indeed rather slow. 


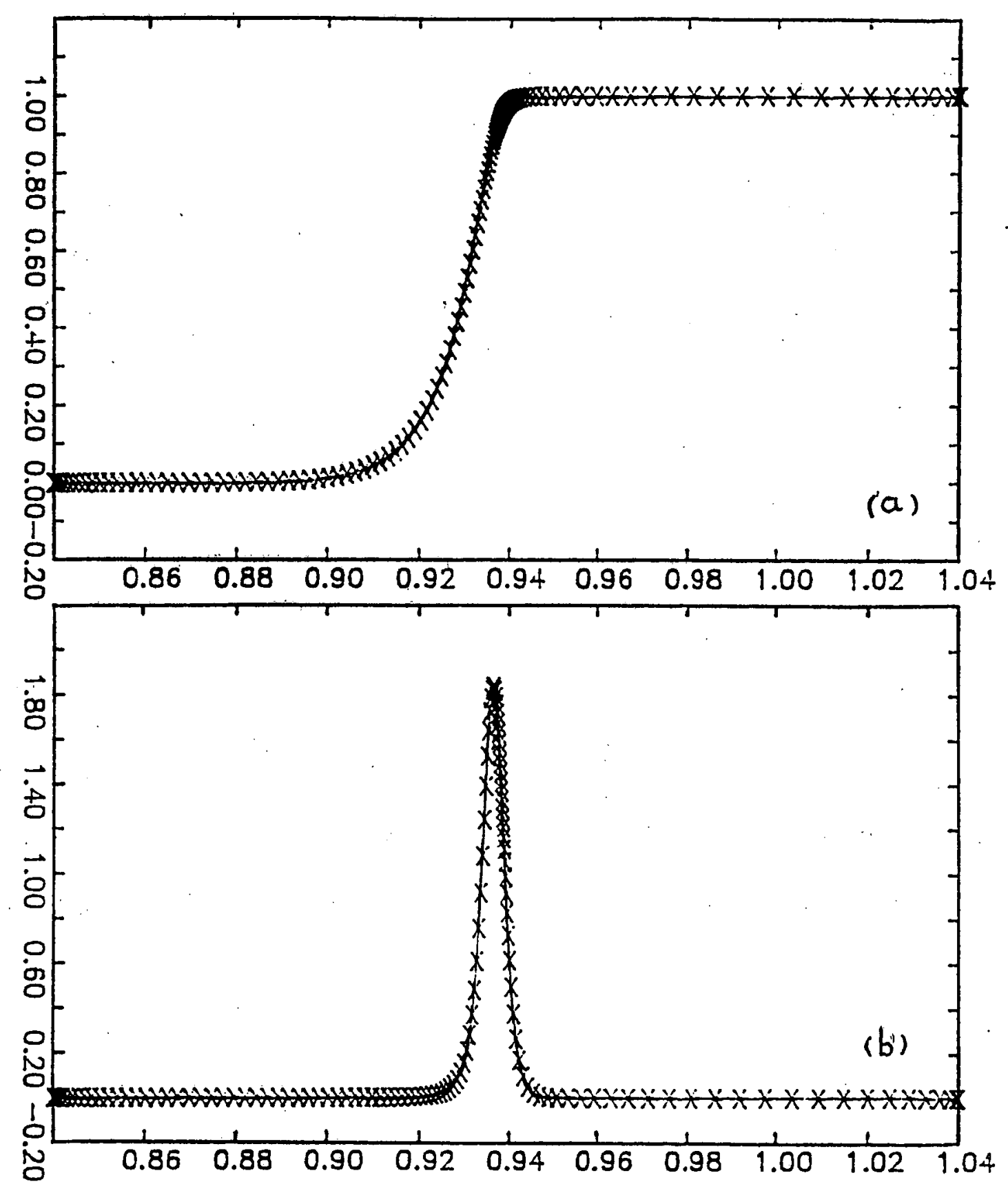

Figure 3.1. Profiles (a) Temperature; (b) reaction rate, obtained with 129 Chebyshev polynomials.

The abscissa must be multiplied by 100 . 
Figure 3.1 presents the temperature and reaction rate profiles of the stationnary solution obtained with 129 Chebyshev polynomials. The convergence curve is displayed on Figure 3.2. Although rather slow the convergence is very regular. After 54000 iterations a residual error of $2.7 \times 10^{-9}$ is reached. This computation requires 2 minutes of CPU time on a cray $1 \mathrm{~S}$ that corresponds to an average of $2.45 \times 10^{-3}$ seconds per iteration. One can see from Figure 3.1 that the collocation points are well distributed and concentrate inside the flame front where the first and second derivatives of the solution are high. The profile of the production term in the computational space is also presented in Figure 3.3. One can observe that the effect of the coordinate tranform is to stretch the flame front and to reduce the magnitude of the derivatives of the solution in the transformed space.

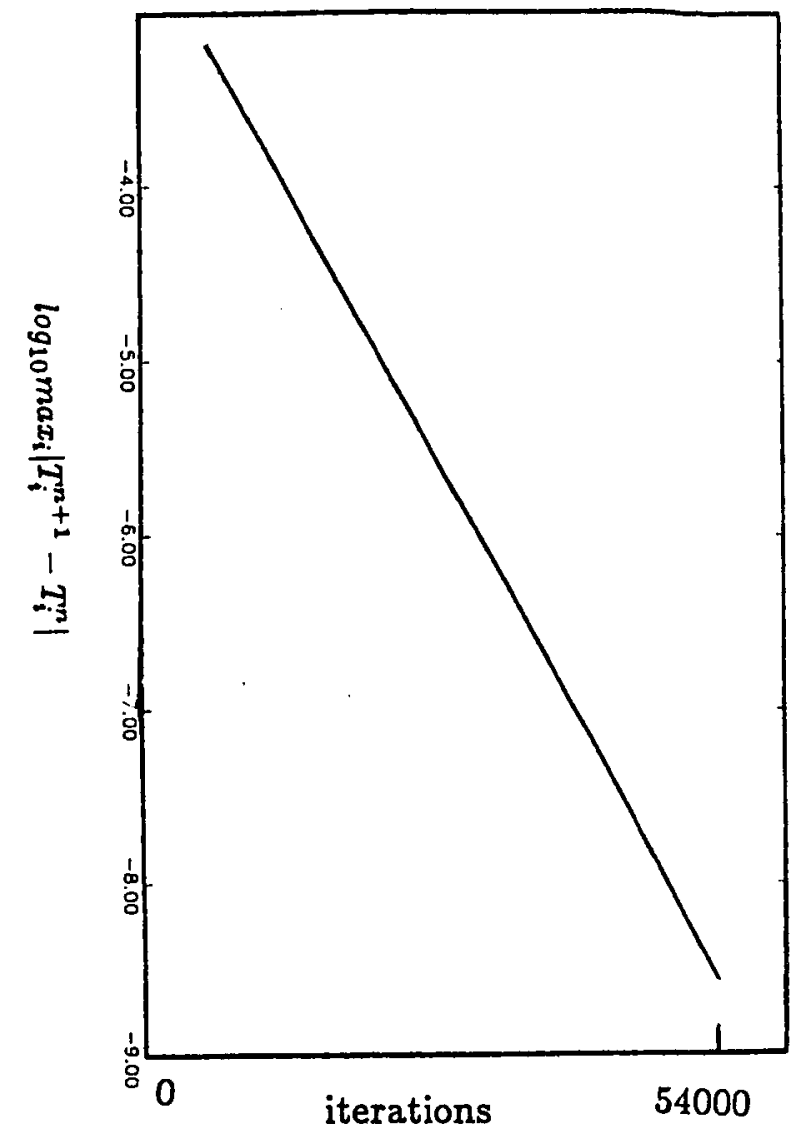

Figure 3.2 : Curve of convergence.

In order to test all the capabilities of the method of coordinate transform we do not performed any filtering of the solution. With 129 Chebyshev polynomials, no oscillations are present in the results. From Figure 3.4 that shows the histogram of the Chebyshev expansion of the solution, we can see that the convergence of this expansion is very good as the last coefficients are of order $10^{-15}$.

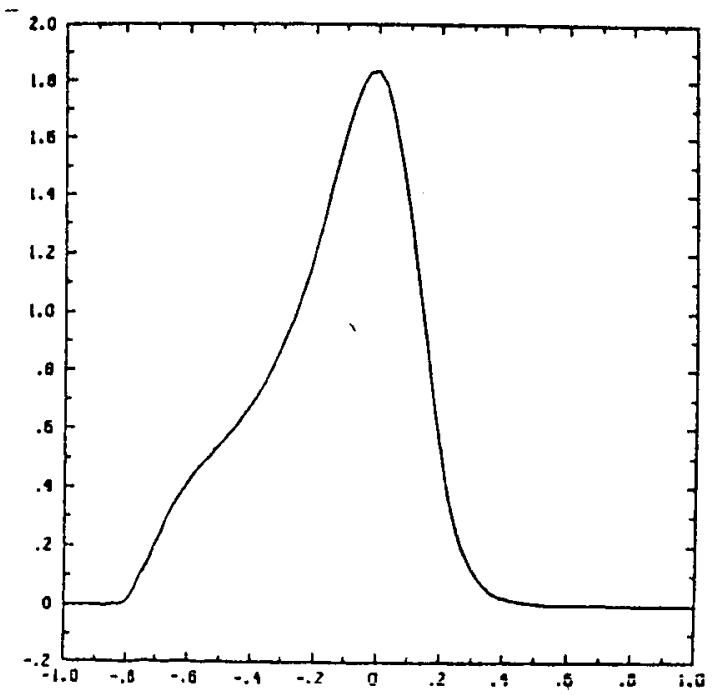

Figure 3.3 : Profile of reaction rate in the computational space. 


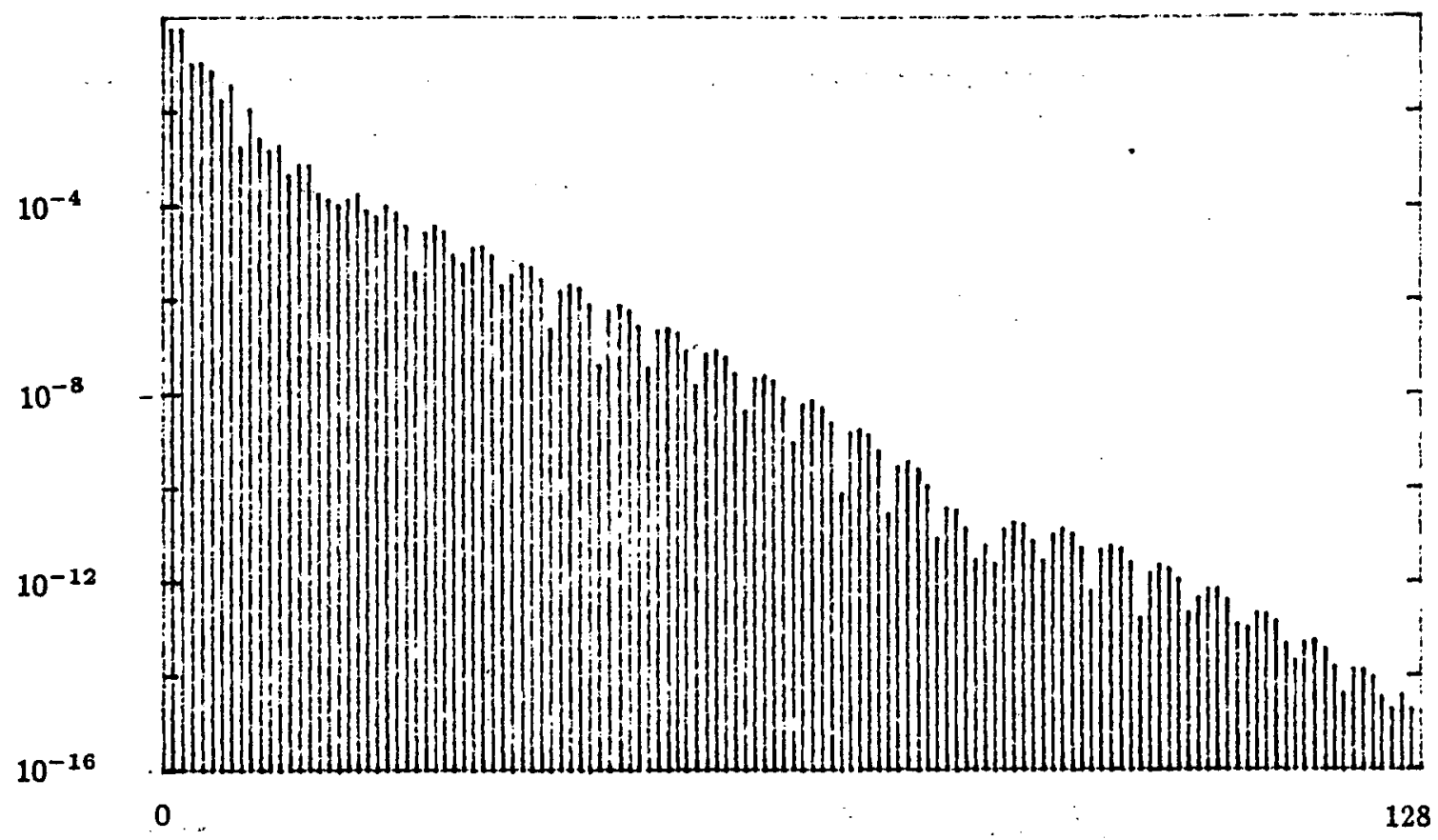

Figure 3.4 : Histogram of the Chebyshev expansion of the stationary solution

To verify the ideas of Section 2, we performed a series of computations where the parameters of the physical problem or of the coordinate transform are modified. An example of these results in wich the value of the mass flux $\kappa$ is slightly modified to 10400 is presented in Figure 3.5. As this mass flux is less important than the first one considered in this Section, the radius of the flame (behaving approximately as $\sqrt{\kappa}$ ) is smaller, and then the coordinate transform is less adapted to this computation. This can be seen in Figure 3.5 where the maximum concentration of the collocation points is downwind the flame front (compare with Figure 3.1.b where the collocation points concentrate inside the flame front). When comparing the norms of these two solutions, the following results are obtained : for the first case $(\kappa=10475)$ we have $\|T\|_{2, \omega}^{2}=12.390$ while for the second case we obtained $\|T\|_{2, \omega}^{2}=26.177$. This confirms the result of Section 2 and shows that even a slight modification of the adaption may lead to a change in the value of the norms by a factor of 2. Therefore the norm of the solution appears as a powerful and objective criterion for adaption. 


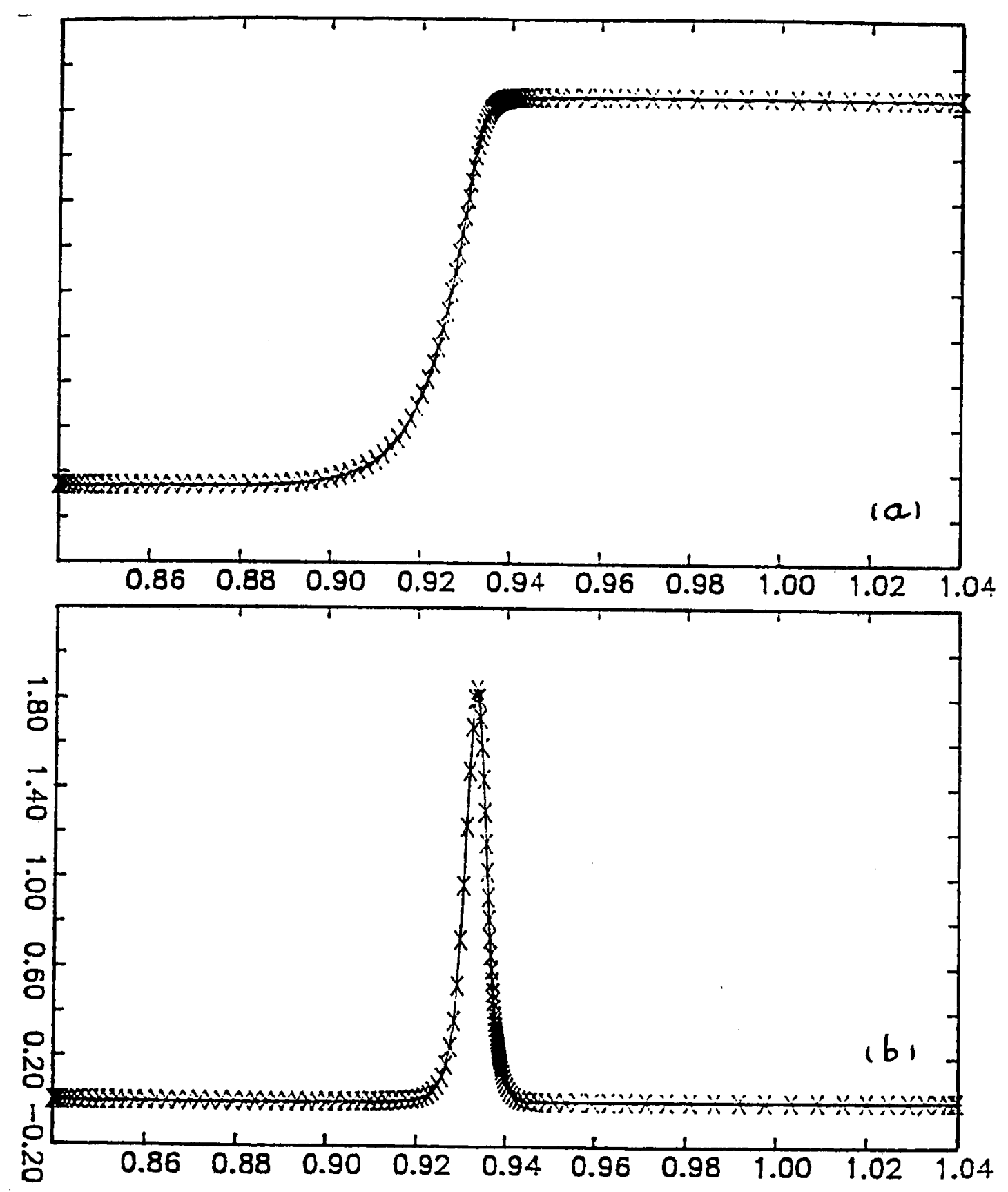

Figure 3.5 : Profiles (a) Temperature; (b) reaction rate, obtained with 129 Chebyshev polynomials.

The abscissa must be multiplied by 100 . 
The same calculation were also done with 65 Chebyshev polynomials. Figure 3.6 illustrates the results corresponding to the same case as Figure 3.1. Although not perceptible at the scale of these pictures, some oscillations (of order $10^{-8}$ ) are present in the temperature and concentration profiles. However, the histogram (Figure 3.7) of the Chebyshev polynomial expansion of the solution indicate no accumulation in the last modes and up to the $65^{\text {th }}$ is almost identical to the one obtained with 129 polynomials. This indicates that the 64 last modes in the 129 polynomial expansion mainly serve to smooth out the oscillations and are not necessary to represent the solution; $A 65$ polynomial expansion with a slight filtering to damp out the oscillation should be sufficient to represent the solution within an accuracy of order $10^{-8}$.

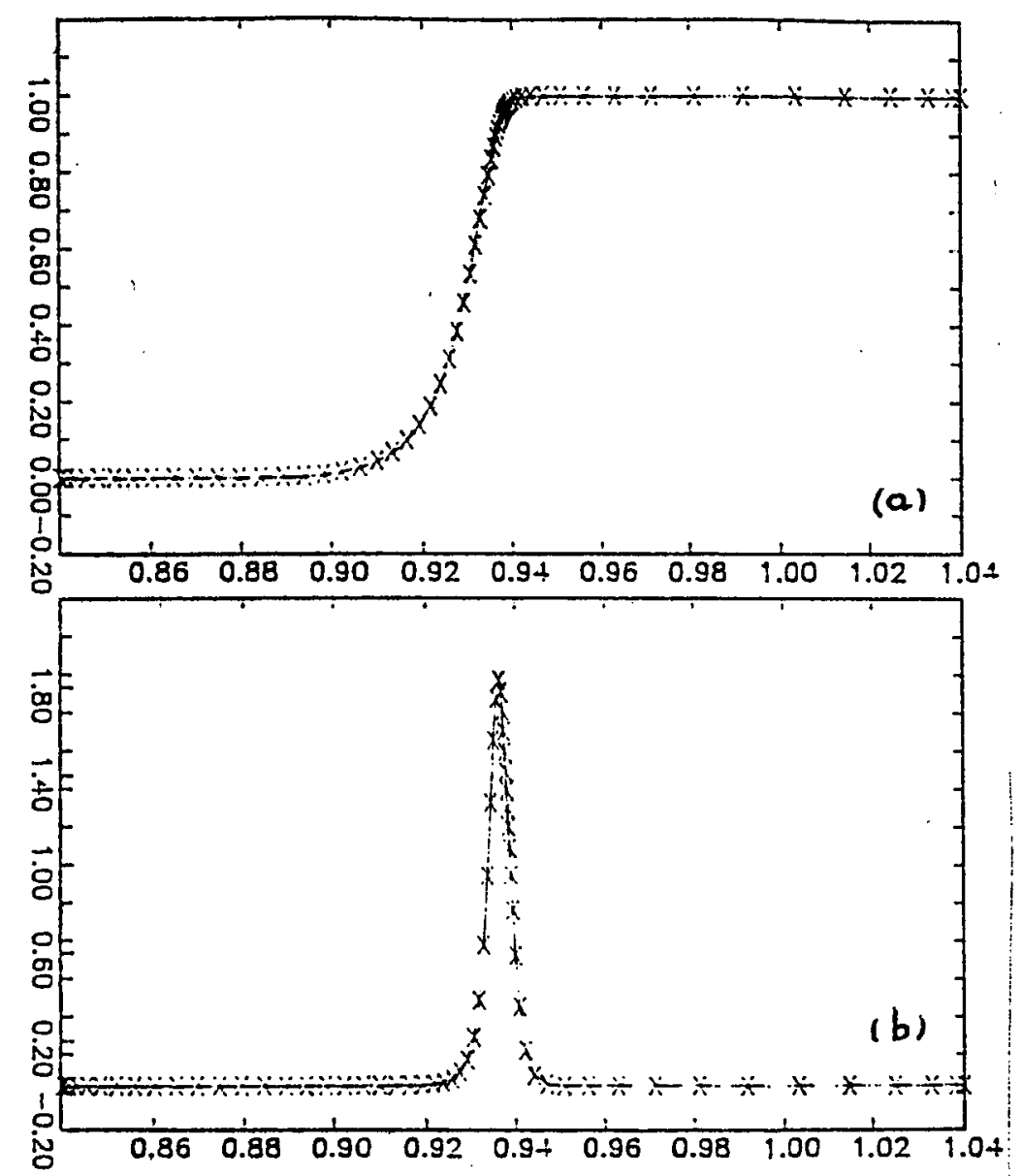

Figure 3.6 : Profiles (a) Temperature; (b) reaction rate, obtained with 65 Chebyshev polynomials.

The abscissa must be multiplied by 100 . 


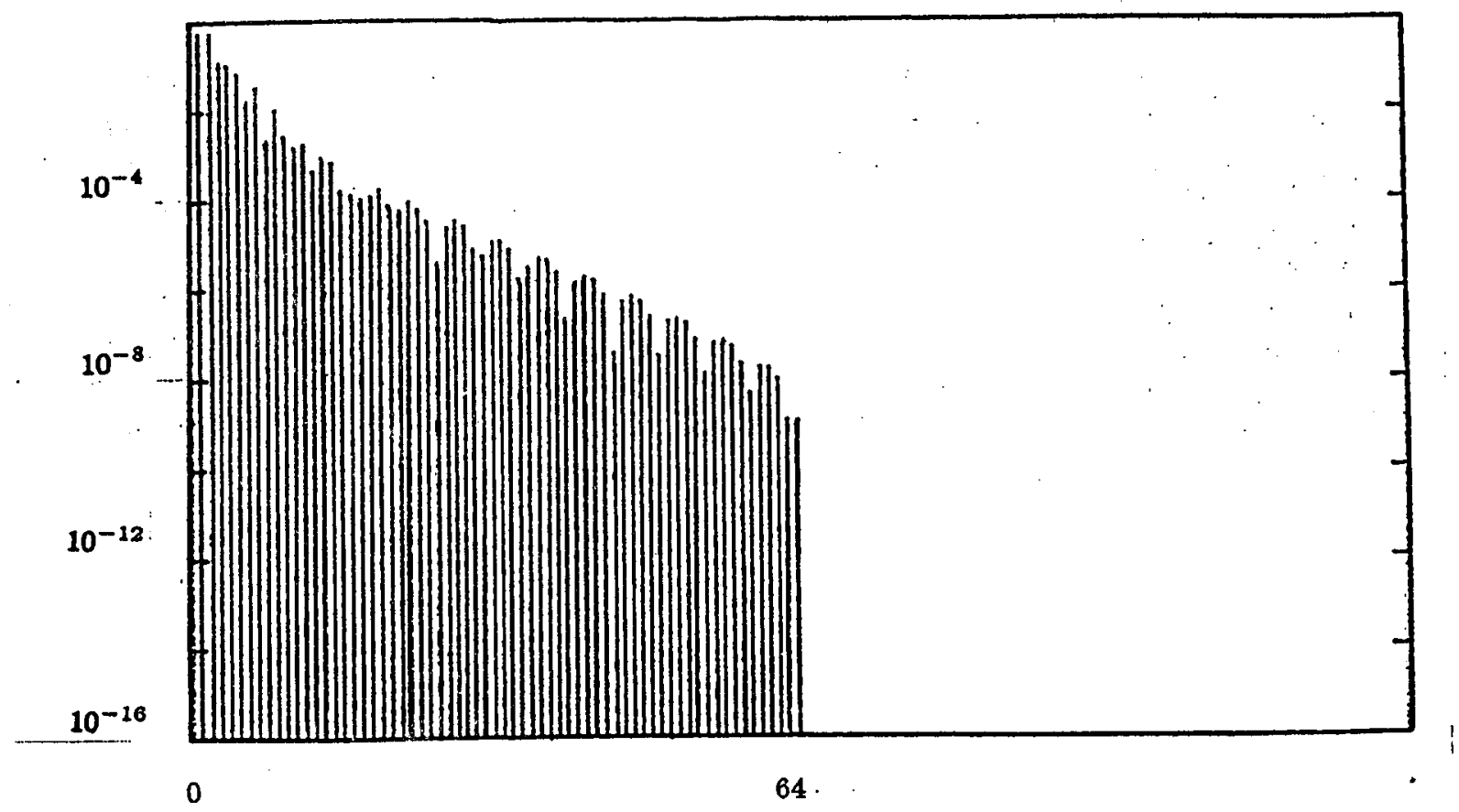

Figure 3.7 : Histogram of the Chebyshev expansion of the stationary solution 


\subsection{Two-dimensional plane flame front.}

\subsubsection{Numerical procedure.}

We consider now, always in the frame of the thermal-diffusional theory of flames, a twodimensional plane flame moving into a reactive medium. The set of equations describing this system writes :

$$
\begin{aligned}
& \frac{\partial C}{\partial t}=\frac{1}{L}\left(\frac{\partial^{2}}{\partial x^{2}} C+\frac{\partial^{2}}{\partial y^{2}} C\right)+\omega \\
& \frac{\partial T}{\partial t}=\frac{\partial^{2}}{\partial x^{2}} T+\frac{\partial^{2}}{\partial y^{2}} T+\omega
\end{aligned}
$$

where $y$ is the direction of propagation of the flame, $x$ the orthogonal direction and the other symbols have the same meaning than in Section 3.2. The boundary conditions are :

$$
\begin{aligned}
& T \rightarrow 0, C \rightarrow 0 \text { as } y \rightarrow-\infty \\
& T \rightarrow 1, C \rightarrow 1 \text { as } y \rightarrow+\infty
\end{aligned}
$$

and $x$ is a direction of periodicity.

The one-dimensional solution of this system is a traveling wave moving at an almost constant speed toward the fresh mixture. Therefore, on a fixed collocation network the flame front will move through it and a fixed coordinate transform such as the one employed in Section 3.2. will be unable to keep a sufficient spatial accuracy in the flame front unless the number of grid nodes will be sufficiently large. To remedy to this situation, the numerical procedure employed in this Section makes use of an adaptive mesh : The collocation network is moved at each time step according to the velocity of the combustion wave, i.e. we introduce the velocities of the collocation points (in this case equal to the same convection velocity $V$ ) and transform the system (3.3.1)-(3.3.2) on a fixed grid to the following system on a moving network :

$$
\begin{aligned}
& \frac{\partial C}{\partial t}-V(t) \frac{\partial}{\partial \eta} C=\frac{1}{L}\left(\frac{\partial^{2}}{\partial \xi^{2}} C+\frac{\partial^{2}}{\partial \eta^{2}} C\right)+\omega \\
& \frac{\partial T}{\partial t}-V(t) \frac{\partial}{\partial \eta} T=\frac{\partial^{2}}{\partial \xi^{2}} T+\frac{\partial^{2}}{\partial \eta^{2}} T+\omega
\end{aligned}
$$


with $x=\xi$ and $y=\eta-V(t) t$. In the present computation, this procedure is combined with a time independent coordinate transform, that concentrates the collocation points in the vicinity of the flame front. So the global coordinate transform applied to (3.3.1)-(3.3.2) has now the form:

$$
\left\{\begin{array}{l}
x=\xi \\
y=g(\eta)-V(t) t
\end{array}\right.
$$

The form of the transform (3.3.7) is precised in appendix II.

When the structure of interest of the solution is simply moving into the computational space (as is the case with waves) this technique may be of great interest. It has been used by some authors with finite difference methods (e.g. Ramos, [27]) and with spectral methods by Hussaini \& Zang [16].

The time discretisation of the system (3.3.5)-(3.3.6) makes use of a factorisation algorithm that solves successively the system in the directions $\xi$ and $\eta$. In the $\xi$-direction a Fourier expansion is used and the computation is performed in the spectral space with a pseudospectral evaluation of the nonlinear production term $\omega$ while in the $\eta$ direction a Chebyshev collocation procedure similar to the one described in Section 3.2 is used. The time scheme is implicit for the diffusion terms of equations (3.3.5) and (3.3.6) while the production and convection terms are evaluated at the previous time step. Therefore the linear systems that are to be solved at each time step, have time independent matrices, this will permit to invert them once for all at the beginning of the calculation.

\subsubsection{Numerical results.}

In the following, we shall consider the problem of the stability of a plane flame front. According to the analysis of Sivashinsky [28], performed in the limit of large energy of activation $(\beta \rightarrow+\infty)$ and for near-equidiffusive flame $(1-L$ of order $1 / \beta)$ the behaviour of a flame front is governed by the bifurcation parameter :

$$
\alpha=\frac{\beta}{2}(1-L) .
$$

The linear stability analysis leads for a pertubation of amplification factor $\gamma$ and of wave number $k$ to the following dispersion relation :

$$
\gamma=(\alpha-1) k^{2}-4 \alpha^{2} k^{4}
$$

that shows that a plane flame front is stable for $\alpha \leq 1$ and can become unstable for a value of $\alpha$ greater than 1. Equation (3.3.8) also shows that there is a range of unstable wave numbers from $k=0$ to $k=k_{0}=\frac{\sqrt{\alpha-1}}{2 \alpha}$ and that the other wave numbers must have decreasing amplitude. The noun-lineâr stability analysis of Sivashinsky performed around 

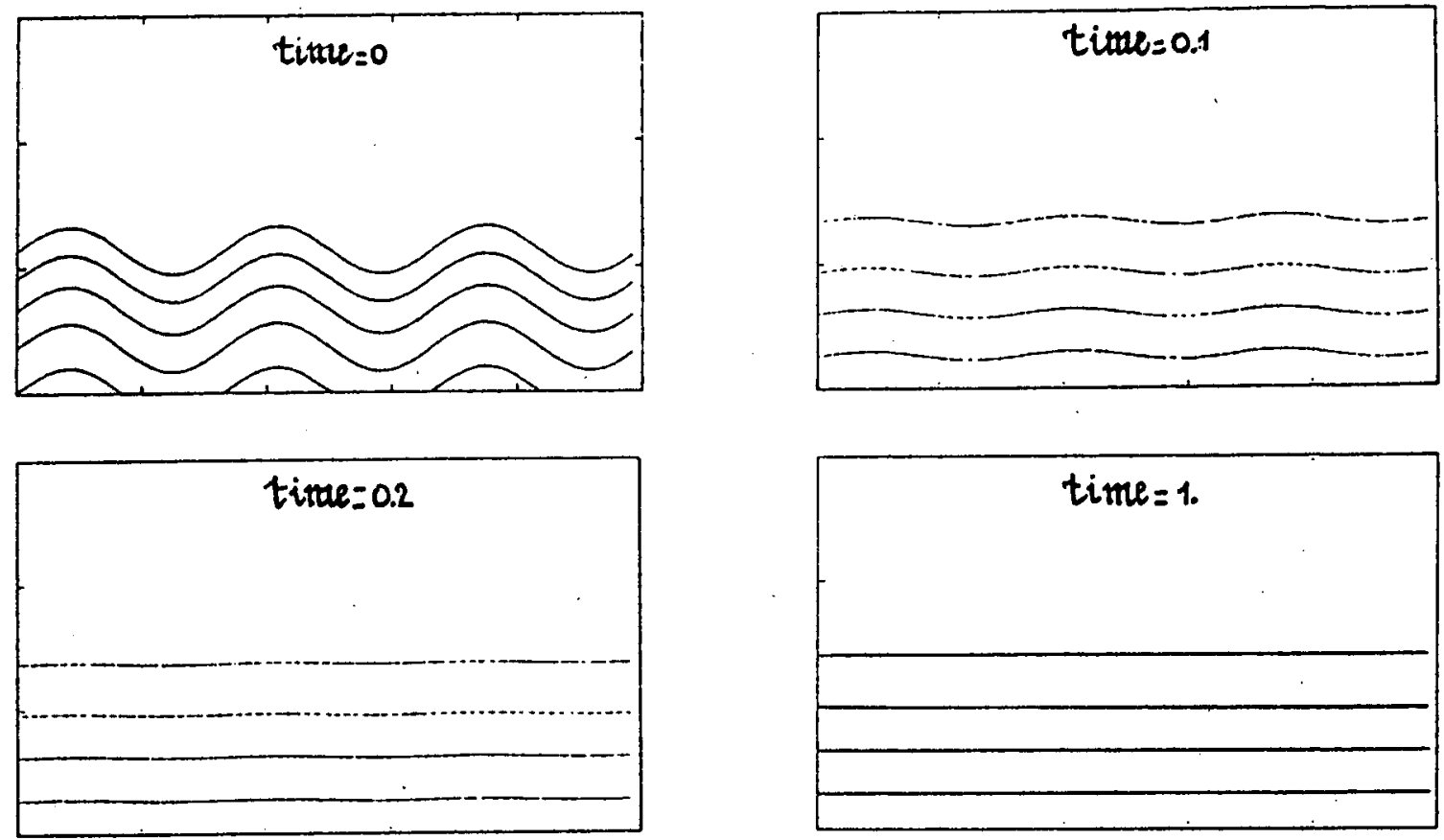

Figure 3.8 : Evolution of isotherms

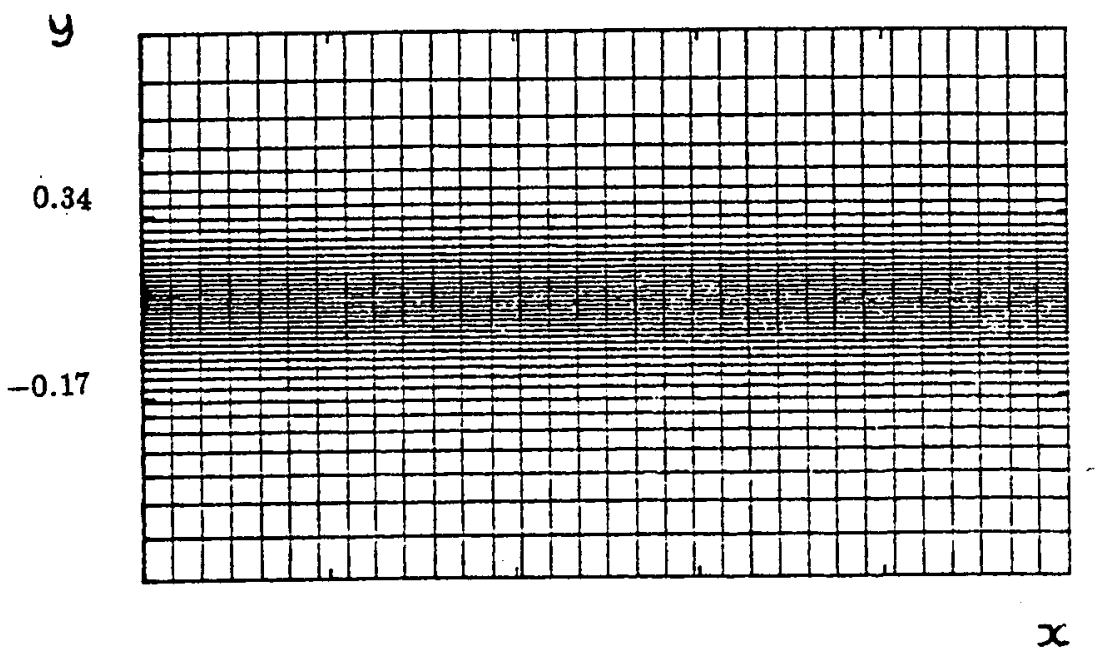

Figure 3.9 : Partial view of the collocation network 
the critical value $\alpha=1$ leads to the well-known Kuramoto-Sivashinsky equation. The numerical studies of this equation show that the behaviour of a flame front evolves to periodic cellular structures and then to a chaotic behaviour as far as the value of $\alpha$ departs from 1 .

\subsubsection{Relaxation of a wrinkled flame to a plane flame.}

In this Section, we study the behaviour of a plane flame front with the following value of the parameters :

$$
\beta=10 ; L=1 \text {. }
$$

In the $\xi$-direction, the dimension of the computational domain is equal to $2 \pi$. A 32 modes Fourier expansion is retained while in the $\eta$ direction a 65 Chebyshev polynomials expansion is used. The initial condition are chosen as the asymptotic steady solution pertubed by a wave of amplitude $a_{p}=0.1$ and of wave-length $\lambda_{p}=2 \pi / 3$. According to the previous stability analysis of Sivashinsky, the amplitude of this pertubation must decrease and the pertubed flame front must relaxed to a plane flame front.

The numerical results are in agreement with the theoretical analysis. Figure 3.8 displays the evolution of the isotherms and shows that the initially pertubed flame front is relaxing toward a plane flame. Figure 3.9 shows that the collocation points concentrate at the average location of the flame front. As the amplitude of the pertubations of the front is small and is decreasing, the spatial resolution of the flame front is sufficient in this case for all $\xi$. This may be seen on Figure 3.10 that displays the production term $\omega$ for different value of $\xi$ at an intermediate time. Figure 3.11 shows the profiles of temperature and reaction rate of the plane flame front obtained when all the pertubations have die out. The coordinate transform is well adapted to this final state and provides a good resolution of the reaction zone. As in the computations of section 3.2 with 65 polynomials, some residual oscillations of order $10^{-8}$ are present in the temperature and concentration profiles. Therefore, as in Section 3.2 these computations would require a slight filtering of the solution to remove these oscillations out.

From the present computation, we conclude that the combination of a time-independent coordinate transform to concentrate the nodes in the region of interest and of a traveling of the reference frame at the wave velocity to follow the displacement of the wave is well designed when the structure of interest of the solution is simply moving in the computational domain and does not experience some fundamental changes. This procedure is in this case very efficient and permits to compute solutions with steep moving gradients with a reasonable computational cost. However, it does not give good results when the structure of the solution is varying as the next computation will show. 


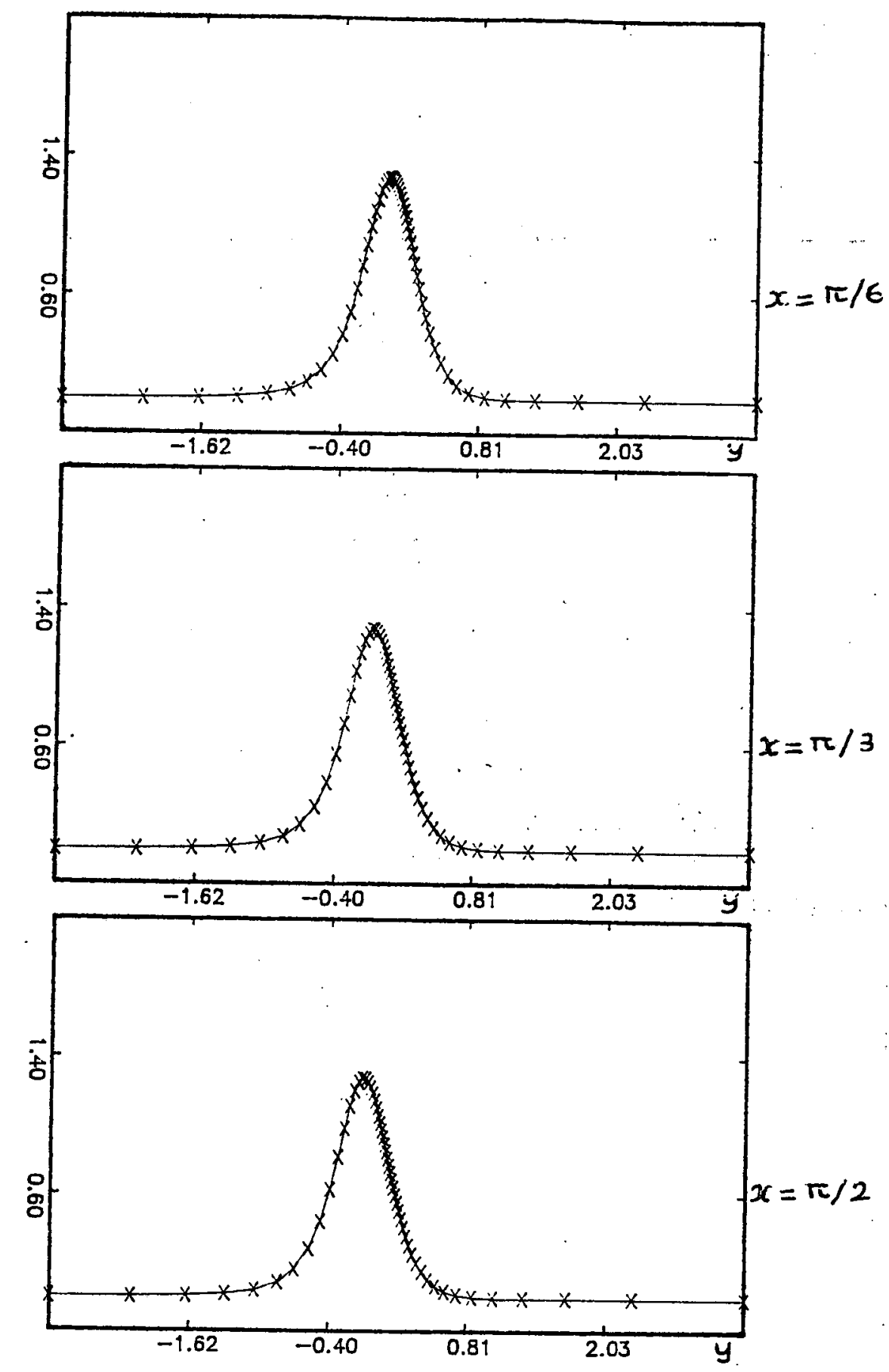

Figure 3.10: Profile of the reaction rate for different value of $x$ 


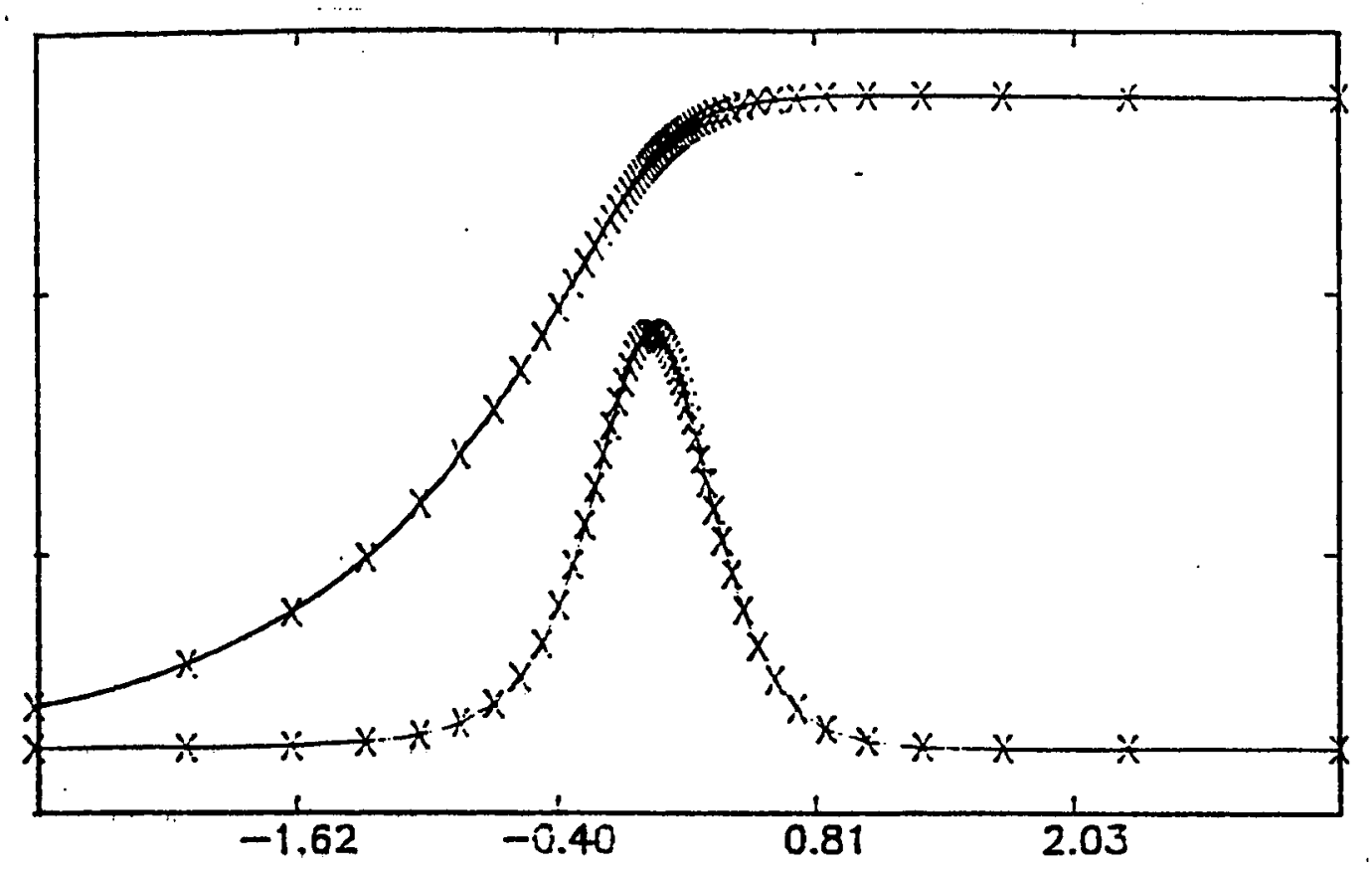

Figure 3.11 : Profiles of temperature and reaction rate of the stationnary solution. 


\subsubsection{Two-dimensional unstable flame front}

We shall, now consider the case of of an unstable flame front. The value of the parameters are:

$$
\beta=10, L=0.7 \text {. }
$$

and the $\xi$-dimension of the computational domain was $3 \lambda_{c}$ where $\lambda_{c}=\frac{4 \sqrt{2} \pi \alpha}{\sqrt{\alpha-1}}$ is the wave-length corresponding to the maximum amplification rate and an initial pertubation of wave-length equal to $\lambda_{c}$ and of amplitude equal to 0.3 was superposed to the basic asymptotic steady solution. As this wave-length is unstable, its amplitude must grow. This is show in Figure 3.12 that displayed the evolution of the isotherms. However, as the amplitude of the pertubation is growing up and the flame front become more and more corrugated, the crests and the troughs of the front go out of the region where the collocation points are concentrated. The spatial resolution of the flame front become insufficient and large oscillations appear in the numerical solution. These oscillations rapidly lead to numerical instabilities and the results of the computation become unreliable. Therefore, the adaptation procedure used in this Section is not suitable for this case. This computation would require a more sophisticated approach in which the coordinate transform would be time-dependent and change according to the evolution of the solution. The following Section will give an example of such a procedure .
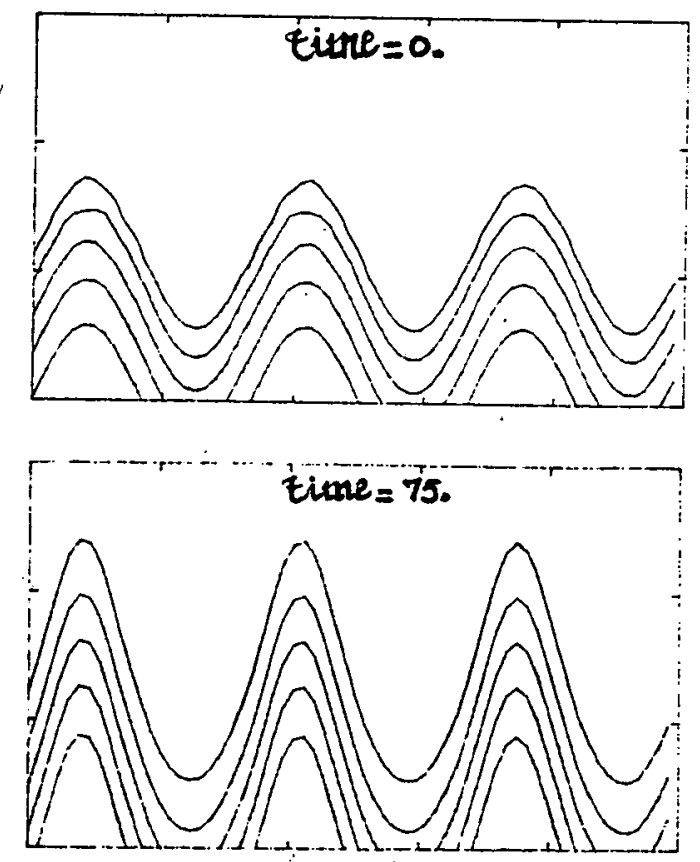

Figure 3.12 : Evolution of the isotherms. 


\subsection{Burgers'equation.}

In this section, we shall deal with the Burgers'equation :

$$
\frac{\partial}{\partial t} u+u \frac{\partial}{\partial x} u=\nu \frac{\partial^{2}}{\partial x^{2}} u,|x| \leq 1, t>0
$$

with the following boundary and initial conditions :

$$
\begin{cases}u( \pm 1, t) & =0, \forall t>0 \\ u(x, 0) & =-\sin \pi x\end{cases}
$$

and the viscosity will be taken to be $\nu=\frac{1}{100 \pi}$. This problem was proposed at a GAMM workshop held in Louvain-la-Neuve at the end of 1980 to investigate the abilities of spectral methods to solve problems whose solutions exhibit sharp variation in a very thin inner layer. Spectral solutions of this problem are presented and compared with finite difference ones in [1] : C. Badesvant has presented a solution using Fourier method, M. Deville and P. Haldenwang used Chebyshev Tau-method and J.M. Lacroix et al applied a Chebyshev collocation procedure while A.T. Patera have used a spectral element method. It was conclude from this investigation that, although not well suited for the computation of thin inner layer, for the same degree of freedom, spectral methods do yield better accuracy than finite difference ones. For the purpose of comparison, we choose to solve this problem with the same numerical method that Lacroix et al: First, a coordinate transform is performed to concentrate the collocation points near the origin and then (3.4.1) becomes :

$$
\frac{\partial}{\partial t} u+\frac{u}{g^{\prime}} \frac{\partial}{\partial \xi} u=\frac{\nu}{g^{\prime 2}}\left(\frac{\partial^{2}}{\partial \xi^{2}} u-\frac{g^{\prime \prime}}{g^{\prime}} \frac{\partial}{\partial \xi} u\right), \quad|\xi| \leq 1
$$

where the coordinate transform $g$ is as in Lacroix et al :

$$
x=g(\xi)=(1-\alpha) \xi^{3}+\alpha \xi, \alpha>0 .
$$

then the spatial part of (3.4.3) is discretized by a Chebyshev collocation procedure where the nonlinear term is evaluated by the pseudo-spectral technique. The time integration makes use of either the $\mathrm{AB} / \mathrm{CN}$ scheme (Adams-Bashforth for the convective part, CrankNicolson for the viscous one) or the AB/2BE scheme (Adams-Bashforth type for the convective part, backward Euler of second order for the viscous one). 
However, unlike Lacroix et al who held fixed in time the coordinate transform $g$, we authorize a time variation of the parameter $\alpha$ and so at each time step we have to choose the value of this parameter. According to the idea exposed in Section 2, this value is choosen to minimize the $H_{\omega}^{2}$ of the computed solution in the computational space. Before going on, note that due to the time dependence of the coordinate transform $g$, equation (3.4.3) would have to be modified by the adjonction at the left hand side of a term coming from the grid speed :

$$
\xi_{t} \frac{\partial}{\partial \xi} u=-\frac{x_{t}}{x_{\xi}} \frac{\partial}{\partial \xi} u
$$

However, following Dwyer \& Sanders [10], we choose to do not evaluate this term and to use the mesh adaptation solely as a regridding procedure. Therefore, we have to interpolate to find the value of the function at the new point locations. That is, we have to compute the term :

$$
u\left(g\left(\xi_{i}\right)\right)=\sum_{n=0}^{N} a_{n} T_{n}\left(g_{o}^{-1}\left(g\left(\xi_{i}\right)\right)\right)
$$

where $g$ is the new coordinate transform, $g_{o}$ the old one and $\sum_{n=0}^{N} a_{n} T_{n}$ is the Chebyshev expansion representing $u\left(g_{o}(\xi)\right)$ This interpolation proceeds as follows : with the known value of the functions $u\left(g_{\circ}(\xi)\right)$ at the collocation points, compute the coefficients $a_{n}$ then compute $g_{o}^{-1}\left(g\left(\xi_{i}\right)\right)$ i.e the inverse image by the old transform in the computational space of the new value of the collocation points in the physical space, and finally by a matrix product compute the term (3.4.6).

Two remarks have to be done about this interpolation : $1^{\text {st }}$ ) we have to invert the coordinate transform $g$, in the present case, it requires to solve a cubic equation and therefore the inversion is exact but it may not be the case in general and numerical inversion may be necessary and $2^{\text {nd }}$ ) during the course of the computation the function defined by (3.4.6) (which is not a polynomial) is replaced by its polynomial interpolation. For these two reasons, the regridding procedure yields an additional spatial error. In the present calculation we expect that this error is small (which is confirmed by the numerical results) but this might be a problem and should require further investigations.

Now, let us recall some characteristics of the solution of (3.4.1). For the small selected value of the viscosity $\left(\nu=\frac{1}{100 \pi}\right)$ the solution develops into a sawtooth wave at the origin from about the time $t=1 / \pi$ and the gradient at the origin reaches its maximum value at about $t=0.5$. This value represents the travel time of the initial velocity peaks of the $u$ profile at $x_{\max }= \pm 0.5$ freesteaming with a velocity $u= \pm 1$ towards the origin. After the maximum value of the gradient at the origin is reached the solution relaxes slowly to zero. Figure 3.13 displaying the development of the $u$ profile obtained with 65 Chebyshev polynomials shows the agreement between the behaviour of the numerical solution and the one previously described. Note that there are no oscillations in this result. 


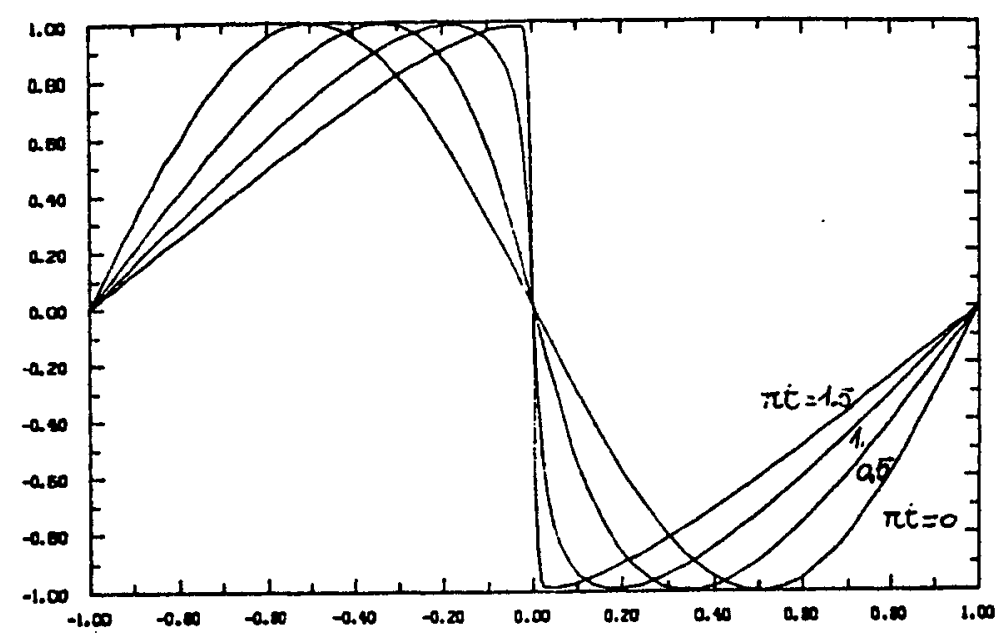

Figure 3.13. Solution at different times.

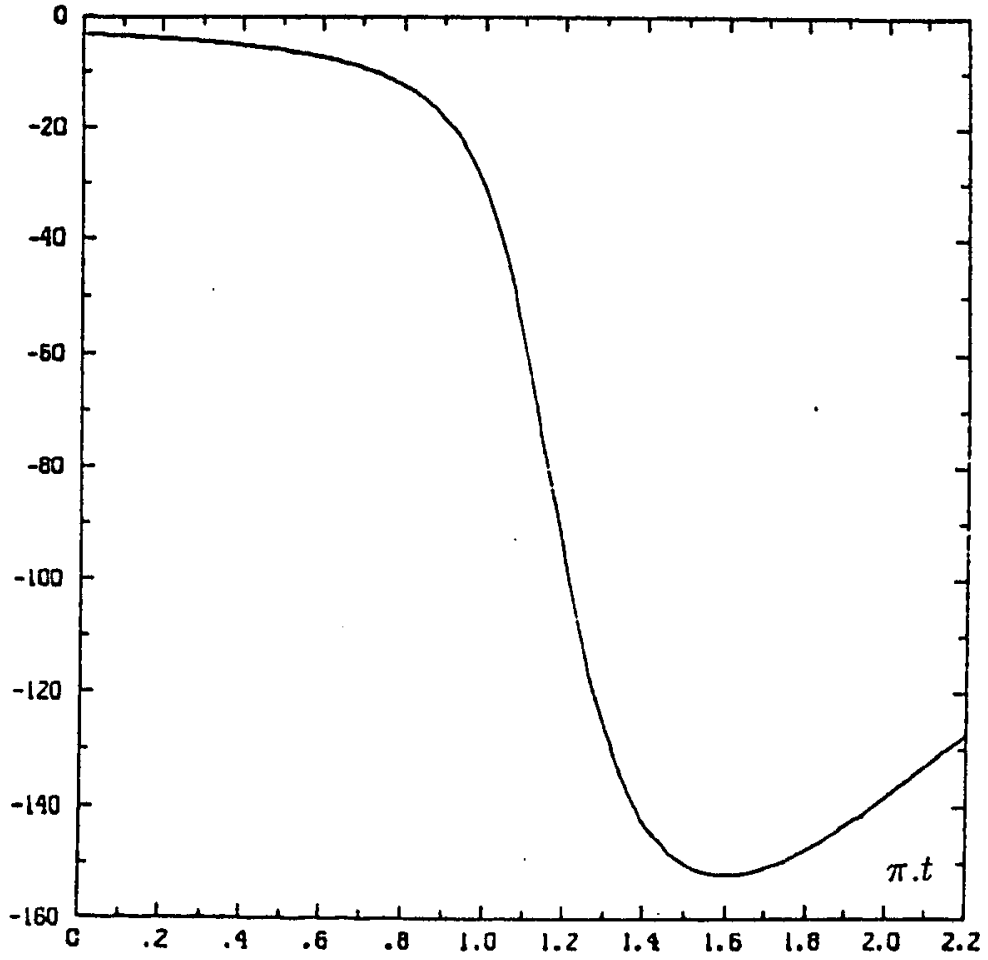

Figure 3.14. Evolution of the value of the slope at the origin versus time 
Figure 3.14 shows the evolution of the slope at the origin. When looking at the maximum value of the gradient at the origin that is a very sensitive value of the precision of the scheme, we obtain :

$$
\left|\frac{\partial}{\partial x} u\right|_{\max }=152.0095 \text { and } \pi \cdot t_{\max }=1.6
$$

These value compare very favorably with the exact ones (obtained from the analytical solution of (3.4.1), see [1]) :

$$
\left|\frac{\partial}{\partial x} u\right|_{\max }=152.00516 \text { and } \pi . t_{\max }=1.6037
$$

and with the numerical ones obtained at the GAMM workshop [1]. Now, let us comment the behaviour of the parameter $\alpha$ of the transform which is displayed Figure 3.15. One can see that at the beginning of the computation, the value of $\alpha$ is close to 1 and then as the large gradient region develops, $\alpha$ decreases to concentrate more and more collocation points in the vicinity of the origin. As the computation proceeds, $\alpha$ becomes smaller and tends to 0 . This lead to the same phenomena observed by Lacroix et al with their fixed coordinate transform : the results were degraded when the density of collocation points near the origin is superior to a certain value. In [1], it was hypothesized that the computation and the inversion of the matrix might be responsible of this fact. However, the same computation performed with an explicit scheme produces identical results. Therefore, the reason is elsewhere: it is possible that the too large concentration of collocation points in the vicinity of the origin leads to a bad representation of the solution outside this region and thus, owing to the non-local character of spectral schemes produces a global decrease of the accuracy. So, we arbitrary limit the decrease of the parameter $\alpha$; Observe that this limitation of the value of $\alpha$ is strictly equivalent to the requirement that the distribution of collocation points in the physical space does not depart too much from the usual Chebyshev one. Difference methods also know this kind of constraint : For example, adaptive methods that distribute the node points according to the minimization of a functional include in the definition of this functional a term leading to a regular grid, (see for example [10],[18] or [3]). The weight of such a term in relation of the other terms of the functional is somewhat arbitrary and to our knowledge, no proposition have been done to give an objective criterion for the choice of such a term. Like Lacroix et al [1], we find that the best value for $\alpha$ is $\alpha=1 / 25$. The results presented above were obtained with this value as a lower bound. 


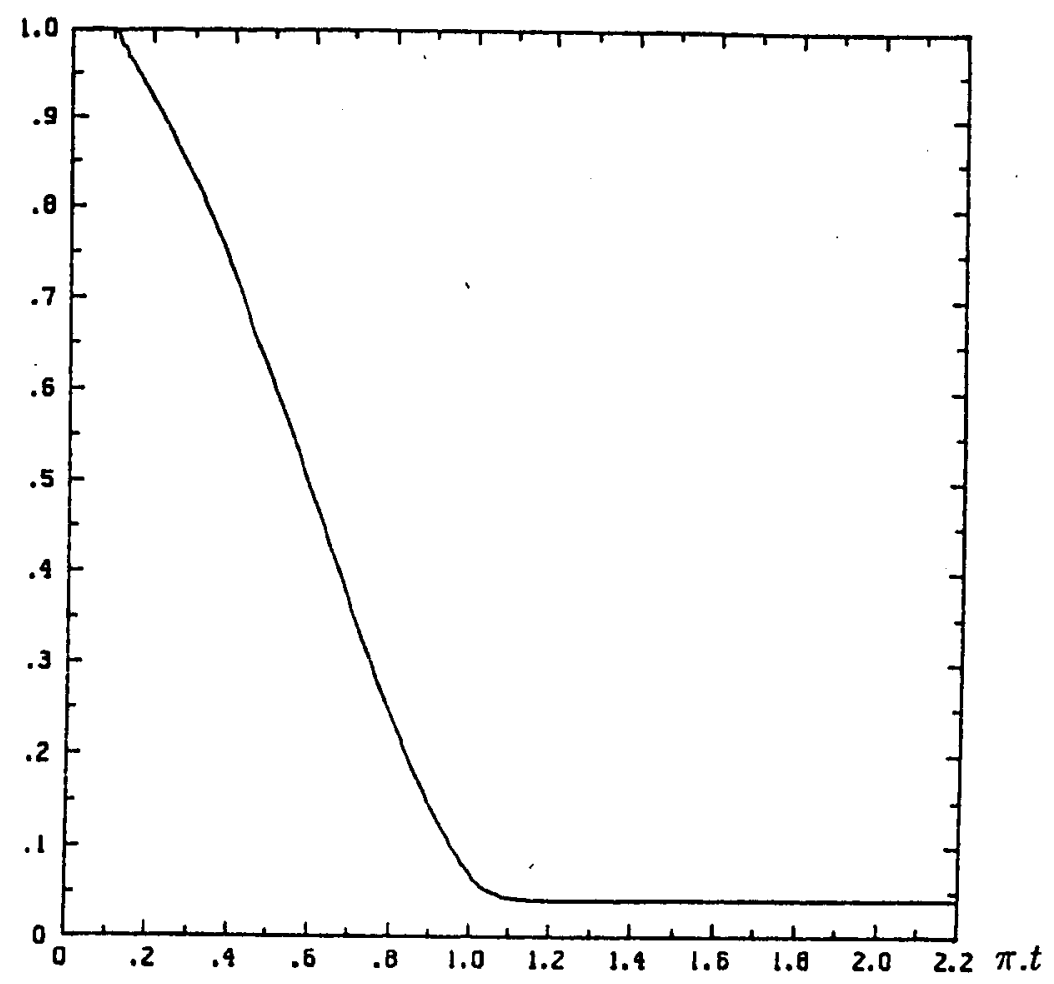

Figure 3.15 Evolution of the parameter $\alpha$ of the coordinate transform.

\section{CONCLUSION}

We investigate a procedure of adaptation suitable for spectral methods. Let us recall that owing to their non-local character, spectral methods do not have the same flexibility than finite difference ones to distribute the nodes points in an arbritrary manner : In the present case of Chebyshev approximation, the appropriate collocation points have to be the zeros or extrema of the higher degree polynomial. Then the distribution of collocation points is imposed. However, as the numerical examples presented in this paper show, the use of coordinate transform may be a partial remedy to this disavantage. The technique of coordinate transform permits both to lower the stiffness of the problem and to keep the accuracy of the spectral calculation. Moreover, the procedure described in Section 2 allows the use of an adaptive coordinate transform in relation to the evolution of the solution.

The computational effort associated with these technique is essentially connected with the time dependency of the coordinate transform.

If the transform is not time dependent, there is no special difficulties to apply the technique of coordinate transform to each problem whose solution is regular. Technically speaking, 
the difficuties are similar to the ones encountered for the matching of complex geometry. Therefore this procedure may be used in conjonction with either an explicit or an implicit scheme and with a truly spectral or a collocation type space discretisation (although the collocation method would be simpler to implement).

However, if a time dependent transform have to be used, the matrices resulting from the space discretisation will be also time dependent, then implicit scheme will require (at least) to solve a different linear system at each time step. As we have verified in the examples presented above, because of the full matrices encountered in spectral methods, this is very time consumming, therefore in our believing the use of time dependent adaptive methods must be nowdays associated with explicit scheme or with mixed difference/spectral methods (spectral approximation for the explicit part of the computation, difference ap-
proximation for the implicit part). 


\section{REFERENCES}

[1] C. BASDEVANT, M. DEVILLE, P. HALDENWANG, J. M. LACROIX, J. OUAZZANI, R. PEYRET, P. ORLANDI, A. T. PATERA, "Spectral and finite difference solutions of the Burgers equation.", Computers $\&$ Fluids, Vol 14, No 1 (1986), pp 23-41.

[2] M. E. BRACHET, "Simulation numérique directe d'écoulements turbulents tridimensionnels.", Thèse d'état. Univ. Nice. June 1983.

[3] J. J BRACKBILL and J. S. SALTZMAN, " Adaptive zoning for singular problems in two dimensions. ", J. Comput. Phys., Vol 46, No. 3 (1982), pp 342-368.

[4] J. P. BOYD, " The optimization of convergence for Chebyshev polynomial method in an unbounded domain.”, J. Comput. Phys., Vol 45 (1982), pp 43-79.

[5] C. CANUTO and A. QUARTERONI, " Approximation results for orthogonal polynomials in Sobolev spaces." Math. Comp., Vol 38, No. 157 (1982), pp 67-86.

[6] C. CANUTO and A. QUARTERONI, "Spectral and pseudo-spectral methods for parabolic problems with non periodic boundary conditions.", Calcolo, Vol. 18 (1981), pp 197-217.

[7] C. CANUTO and A. QUARTERONI, "Variational methods in the theoretical analysis of spectral approximations.", Proceeding of the Worshop on Spectral methods (Hampton, Virginia, August 1982), (Gottlieb, Hussaini \& Voigt Eds), (1984), SIAM, Philadelphia.

[8] B. DESHAIES, G. JOULIN and P. CLAVIN, " Etude asymptotiques des flammes sphériques non adiabatiques.", Journal de Mécanique, Vol 20., No. 4 (1981), pp 691-735.

[9] G. DURAND, "Etude de l'approximation par interpolation polynomiale et des algorithmes de transformée de Fourier rapide : Application à la méthode spectrale de collocation.", Thèse de Troisième Cycle. Dpt. Math, Univ. Nice, July 1985.

[10] H. A. DWYER and B. R. SANDERS, "Numerical modelling of unsteady flame propation. ", Acta Astronautica Vol 5,(1978), pp 1171-1184.

[11] B. FORNBERG, " On a Fourier method for the integration of hyperbolic equations.", SIAMÍ J. Numer. Anai., Voil 12, (1975), pp 509-528. 
[12] R. J. GELINAS, S. K. DOSS and K. MILLER, "The moving finite element method: Applications to general partial differential equations with multiple large gradients.", $J$. Comput. Phys., Vol 40 (1981), pp 202-249.

[13] D. GOTTLIEB and L. LUSTMAN, "The spectrum of the Chebyshev collocation operator for the heat equation.”, SIAM J. Numer. Anal., Vol 20, No. 5, (1983), pp 909-921.

[14] D. GOTTLIEB and S. A. ORSZAG, "Numerical analysis of spectral methods : Theory and application.",CBMS Regional Conference Series in Applied Mathematics 26, SIAM, Philadelphia,(1977).

[15] C. E. GROSH and S. A. ORSZAG, "Numerical solution of problems in unbounded regions : coordinate transforms. ", J. Comput. Phys., Vol 25, (1977), pp 273-296.

[16] N. Y. HUSSAINI and T. A. ZANG, "iterative spectral methods and spectral solutions to compressible flows." ICASE Report No. 82-40, November 1982, NASA Langley Research Center, Hampton, Virginia 23665.

[17] H. O. KREISS and J. OLIGER, " Stability of the Fourier method.", SIAM J.1Numer. Anal., Vol 16, No. 3 (1979), pp 421-433.

[18] B. LARROUTUROU, "Adaptive numerical methods for unsteady flame propagation.", Proceedings of the 1985 AMS-SIAM Summer Seminar on "Reacting flows: Combustion and chemical reactors", Ludford Ed.,to appear.

[19] Y. MADAY and A. QUARTERONI, " Legendre and Chebyshev spectral approximations of Burgers'equation.", Numer. Math, 37 (1981), pp 321-332.

[20] B. MERCIER, "Analyse numérique des méthodes spectrales.", Note CEA No. 2278, June 1981, CEA, Centre d'etudes de Limeil, 94190 Villeneuve-Saint-Georges.

[21] K. MILLER and R. N. MILLER, "Moving finite elements.", SIAM J. Numer. Anal., Vol 18, No. 6 (1981),pp 1019-1032.

[22] K. MILLER, "Moving finite elements.", SIAM J. Numer. Anal., Vol 18, No. 6 (1981), pp 1033-1057.

[23] Y. MORCHOISNE, " Subdomain spectral method for Navier-Stokes equations resolution.", Proceeding of the third international symposium on numerical methods in engineering.",(Lascaux Ed), March 1983,Paris.

[24] S. A. ORSZAG,"Spectral methods for problems in complex geometries.",J. Comput. Phys., Vol 40,(1981), pp 70-92. 
[25] J. OUAZZANI and R. PEYRET, "A pseudo-spectral solution of binary gas mixture flows." Notes on Numerical Fluids Mechanics (Pandolfi \& Piva Eds), Vol 7 (1984), pp 275-282. Vieweg Verlag, Braunschweig.

[26] J. E. PASCIAK, "Spectral and pseudo-spectral methods for advection equations.", Math. Comp., Vol 35, No. 152 (1980), pp 1081-1092.

[27] J. I. RAMOS, "The application of finite difference and finite element methods to a reaction-diffusion system in combustion" in Numerical methods in laminar and turbulent flows.,(Taylor,Johnson and Smith, Eds) , 1983, Pineridge Press, Swansea, UK, pp 11371147.

[28] G. I. SIVASHINSKY, " Nonlinear analysis of hydrodynamic instability in laminar flame - 1. Derivation of basic equations.”, Acta Astronautica. , Vol 4 (1977), pp 11771206.

[29] G. I. SIVASHINSKY, " On self-turbulization of a laminar flame.",Acta Astronautica. , Vol 6 (1979), pp 569-591.

[30] J. F. THOMPSON, "Grid generation techniques in computational fluid dynamics.", AIAA Journal, Vol 22,(1984), pp 1505-1523. 


\section{APPENDIX I}

Definition of the coordinate transform (3.2.6)

$$
\begin{aligned}
& {[-1,+1] \longrightarrow\left[r_{0}, r_{\infty}\right]} \\
& \xi \stackrel{\mathrm{g}}{\longrightarrow} r=a+b e^{-\lambda \xi}-c e^{\nu \xi}
\end{aligned}
$$

$\lambda$ and $\nu$ are arbitrary positive parameters.

$a, b$ and $c$ are determined such that :

$$
g(+1)=r_{0}, \quad g(-1)=r_{\infty} \text { and } g\left(\xi_{0}\right)=r_{f}\left(r_{0}<r_{f}<r_{\infty}\right)
$$

where $\xi_{0}$ that corresponds to the location of the flame front at initial time, may be chosen arbitrary in $[-1,+1]$. Numerical experiments show that best results are obtained when $-1<\xi_{0}<0$.

The condition A.1 leads to :

$$
\begin{gathered}
c=\frac{C\left(r_{\infty}-r_{0}\right)+A\left(r_{f}-r_{0}\right)}{A D-B C} ; b=\frac{D\left(r_{\infty}-r_{f}\right)+B\left(r_{f}-r_{0}\right)}{A D-B C} \\
a=r_{\infty}-b e^{\lambda}+c e^{-\nu}
\end{gathered}
$$

with:

$$
\begin{aligned}
& A=e^{\lambda}-e^{-\lambda \xi_{0}} \quad ; B=e^{-\nu}-e^{\nu \xi_{0}} \\
& C=e^{-\lambda}-e^{-\lambda \xi_{0}} \quad ; D=e^{\nu}-e^{\nu \xi_{0}}
\end{aligned}
$$

We want $g^{\prime}\left(\xi_{0}\right)$ sufficiently small in order to have more points in the front region, from the above expressions we get:

$$
g^{\prime}\left(\xi_{0}\right) \sim-\lambda\left(r_{\infty}-r_{0}\right) e^{-\lambda\left(\xi_{0}+1\right)}-\nu\left(r_{f}-r_{0}\right) e^{\nu\left(\xi_{0}-1\right)}
$$

if $\lambda \gg 1$ and $\nu \gg 1$.

In the computations presented above, we use: $r_{0}=r_{f}-10, r_{f}=94, r_{\infty}=r_{f}+10, \xi_{0}=$ $-0.7, \lambda=15$ and $\nu=3$. 


\section{APPENDIX II}

Definition of the coordinate transform (3.3.7)

$$
\left\{\begin{array}{l}
x=\xi \\
y=g(\eta)-V(t) t
\end{array}\right.
$$

The velocity $V$ of the flame is defined as :

$$
V=\frac{1}{H} \int_{-\infty}^{+\infty} \int_{0}^{H} \omega d x d y
$$

and the function $g$ maps $[-1,+1]$ into $]-\infty,+\infty[$. This function has the following form:

$$
g(\eta)=a 1+\frac{a 2}{1-\eta}-\frac{a 3}{1+\eta}
$$

where the constant $a 2, a 3$ are defined by :

$$
a 2=\frac{a}{2}\left(1-\eta_{0}^{2}\right)\left(1-\eta_{0}\right) \text { and } a 3=\frac{a}{2}\left(1-\eta_{0}^{2}\right)\left(1+\eta_{0}\right)
$$

so that for $\eta=\eta_{0}, g^{\prime}\left(\eta_{0}\right)=a$. The following value of the parameters are used in the computations presented above :

$$
a 1=0.3, \quad a=0.3 \text { and } \eta_{0}=0.3
$$


